Preprint, for published version see Combustion and Flame 167:24-38, 2016. http://dx.doi.org/10.1016/j.combustflame.2016.02.030.

\title{
Detonation Mode and Frequency Analysis Under High Loss Conditions for Stoichiometric Propane-Oxygen
}

\author{
Scott Jackson ${ }^{1, *}$, Bok Jik Lee ${ }^{2}$, Joseph E. Shepherd ${ }^{3}$ \\ Graduate Aeronautical Laboratories \\ California Institute of Technology, Pasadena, CA 91125
}

\begin{abstract}
The propagation characteristics of galloping detonations were quantified with a high-time-resolution velocity diagnostic. Combustion waves were initiated in 30-m lengths of 4.1-mm inner diameter transparent tubing filled with stoichiometric propane-oxygen mixtures. Chemiluminescence from the resulting waves was imaged to determine the luminous wave front position and velocity every 83.3 microseconds. As the mixture initial pressure was decreased from 20 to $7 \mathrm{kPa}$, the wave was observed to become increasingly unsteady and transition from steady detonation to a galloping detonation. While wave velocities averaged over the full tube length smoothly decreased with initial pressure down to half of the ChapmanJouguet detonation velocity $\left(D_{\mathrm{CJ}}\right)$ at the quenching limit, the actual propagation mechanism was seen to be a galloping wave with a cycle period of approximately $1.0 \mathrm{~ms}$, corresponding to a cycle length of $1.3-2.0 \mathrm{~m}$ or $317-488$ tube diameters depending on the average wave speed. The long test section length of 7,300 tube diameters allowed observation of up to 20 galloping cycles, allowing for statisti-

\footnotetext{
*Corresponding author

Email addresses: s jackson@lanl.gov (Scott Jackson), BokJik. Lee@kaust. edu.sa (Bok Jik Lee), jeshep@caltech. edu (Joseph E. Shepherd)

${ }^{1}$ Team Leader, Shock and Detonation Physics Group, Los Alamos National Laboratory, Los Alamos, NM 87545 USA

${ }^{2}$ Research Scientist, Clean Combustion Research Center, King Abdullah University of Science and Technology, Thuwal, Saudi Arabia

${ }^{3}$ Professor, Graduate Aeronautical Laboratories, California Institute of Technology, Pasadena, CA 91125 USA
} 
cal analysis of the wave dynamics. In the galloping regime, a bimodal velocity distribution was observed with peaks centered near $0.4 D_{\mathrm{CJ}}$ and $0.95 D_{\mathrm{CJ}}$. Decreasing initial pressure increasingly favored the low velocity mode. Galloping frequencies ranged from 0.8 to $1.0 \mathrm{kHz}$ and were insensitive to initial mixture pressure. Wave deflagration-to-detonation transition and detonation failure trajectories were found to be repeatable in a given test and also across different initial mixture pressures. The temporal duration of wave dwell at the low and high velocity modes during galloping was also quantified. It was found that the mean wave dwell duration in the low velocity mode was a weak function of initial mixture pressure, while the mean dwell time in the high velocity mode depended exponentially on initial mixture pressure. Analysis of the velocity histories using dynamical systems ideas demonstrated trajectories that varied from stable to limit cycles to aperiodic motion with decreasing initial pressure. The results indicate that galloping detonation is a persistent phenomenon at long tube lengths.

Keywords: detonation, DDT, detonation failure, galloping detonation, near limit detonation

\section{Introduction}

Detonation in tubing with diameters approaching the detonation reaction zone length has been shown to be capable of propagating at average velocities that are significantly below the Chapman-Jouguet (CJ) velocity that occurs in largerdiameter tubing. Prior studies have shown a smooth decrease in average detonation velocity in small diameter tubing with decreasing initial pressure $P_{0}$ for stoichiometric propane-oxygen, reaching velocities as low as $0.5 D_{\mathrm{CJ}}$, where $D_{\mathrm{CJ}}$ is the Chapman-Jouguet detonation velocity, before the tube quenching limit was reached (Figure 1). This phenomenon has been the subject of considerable interest [1-5] for many decades with recent literature reviewed by Jackson [6] and Camargo et al. [7].

Some earlier efforts have used microwave interferometry to obtain high-resolution detonation velocity histories of these near-limit detonations $[2,4,10]$. Lee et al. [2] processed such velocity histories to obtain histograms that quantitatively described six detonation modes as mixtures approached the failure limit. As initial mixture pressure decreased for a given tube diameter $d$, self-sustained detonations would transition to detonations with velocity fluctuations. These fluctuations were initially small in magnitude, resulting in unstable waves with instantaneous speeds of $0.7-0.9 D_{\mathrm{CJ}}$. The result of further pressure decreases was mixture dependent 


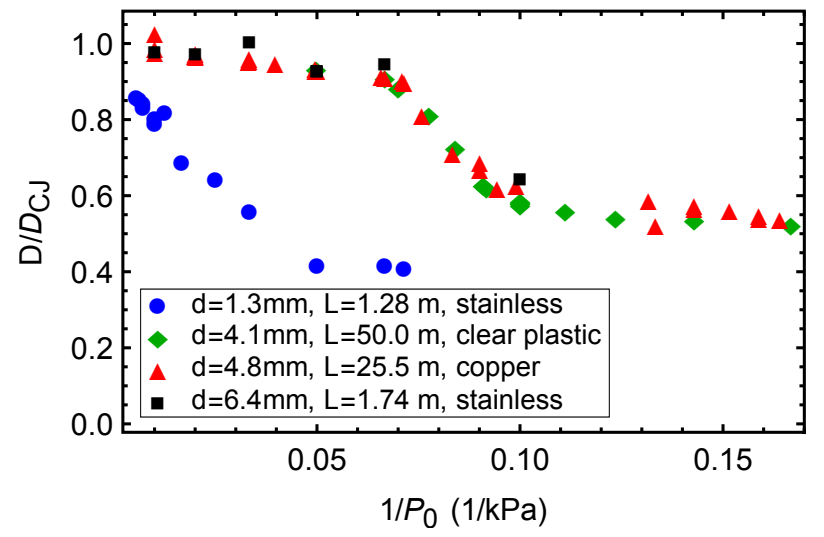

Figure 1: Average combustion wave velocity data for $\mathrm{C}_{3} \mathrm{H}_{8}+5 \mathrm{O}_{2}$ versus inverse pressure for different tube diameters; stainless steel tube data is from Ref. 6, the copper data is from Ref. 8 as reported by Ref. 9, and the clear plastic tubing data is that discussed in the present paper. The test geometries for the 1.3-mm and 6.4-mm diameter tests used straight tube lengths, while the tubing was formed into spirals for the 4.1-mm and 4.8-mm tests.

and attributed to the relative stability [11] of each mixture tested [2]. The effective activation energy, denoted by $\theta$, is often used to quantify a mixture's detonation stability [12]. Mixtures with higher values of $\theta$ generally exhibit more irregular cellular structure and detonation velocity fluctuations near failure. Lee et al. [2] found that, for mixtures with high effective activation energies, lowering mixture pressures could result in the onset of three additional modes: (1) galloping detonations with instantaneous wave speeds between 0.4 and $1.5 D_{\mathrm{CJ}}$, followed by (2) a purely deflagrative mode propagating near $0.4 D_{\mathrm{CJ}}$, followed by (3) reaction quenching. Mixtures with lower effective activation energies did not exhibit the galloping or deflagrative modes and instead would only quench upon further pressure decreases. Their results were likely geometry specific as the detonation velocity in these near-limit detonations is expected to depend on the coupling between the mixture chemical kinetics and the gas-dynamic response to any confinement (in the form of momentum and thermal boundary losses).

The extremely long length of the galloping cycle has made its characterization difficult. Edwards et al. [10] observed up to four galloping cycles in a tube of rectangular cross section over a distance of $20 \mathrm{~m}(L / d=870)$. Lee et al. [2] were not able to observe multiple galloping cycles in their 10-m $(L / d=260)$ tube and noted that "an ambiguity in the identification of the galloping mode and the failure mode may exist" due to this limitation. Haloua et al. [4] were similarly not able 
to observe more than two cycles in their $25-\mathrm{m}(L / d=645)$ tube. More recently, Wu and Wang [13] inferred two galloping cycles from high-speed cinematography in a 1,500-diameter long tube, but with camera sensitivity that was only able to register luminosity during the peak velocity phase. Subsequently, Gao et al. [5] obtained up to five galloping cycles in tubes as long as 1,625 diameters, but with spatially discrete diagnostics. Thus, high-resolution observations of the full galloping cycle have been limited, with little opportunity to study its long-term evolution to determine if the mode is independent of initial ignition conditions, repeatable, and persistent over long times.

In this work, we use a novel, transparent, and spiral tube geometry with highspeed video to obtain high-temporal-resolution velocity measurements of the luminous front present in galloping detonations over distances of $30 \mathrm{~m}(L / d>$ $7,300)$ in stoichiometric propane-oxygen mixtures from 7 to $20 \mathrm{kPa}$. Over the tested pressure range, this mixture has a high effective activation energy $(\theta \approx$ 11 from Schultz and Shepherd [12]) and is considered to be highly unstable with detonation cell sizes ranging from $\lambda=19$ to $5.6 \mathrm{~mm}(\lambda / d=4.6$ to 1.4$)$ for the pressure range of 7 to $20 \mathrm{kPa}$ [14]. The initial mixture pressure $P_{0}$ was varied to obtain different detonation propagation modes. The observation length is sufficiently long to allow for measurement of up to 20 galloping cycles per test, allowing for quantitative and statistical analysis of the galloping phenomenon. Velocity-time profiles of galloping detonation are presented as a function of mixture pressure. Histograms are used to quantify the velocity probability at each test condition. These results are then interpolated to form a velocity probability map versus initial mixture pressure. A map of galloping frequency versus initial pressure is also reported. The timing and repeatability associated with the individual components of the galloping cycle are analyzed. Finally, the stability of the longitudinal velocity pulsations was analyzed and compared to results from one-dimensional detonation calculations.

We emphasize that our measurement technique records only the position and velocity of the luminous front associated with the combustion, in similar fashion to works which use photodiode sensors. We do not measure the position of the leading shock wave, which is commonly reported by numerical simulations, by pressure transducers and by schlieren measurements. In contrast, microwave interferometry studies report the velocity of the ionization front associated with a combustion event or dissociation behind a strong shock. Each of these features may exhibit different dynamics as the shock decouples and recouples with the reaction zone in the unsteady galloping regime. 

sure. A schematic of the experimental setup is shown in Fig. 2. A deflagration

Combustion waves were propagated though small-diameter, transparent, polyurethane tubing filled with stoichiometric propane-oxygen mixtures of varying initial pres-

\section{Experiment}

$$
\text { sure. A schematic of the experimental setup is shown in Fig. 2. A deflagration }
$$

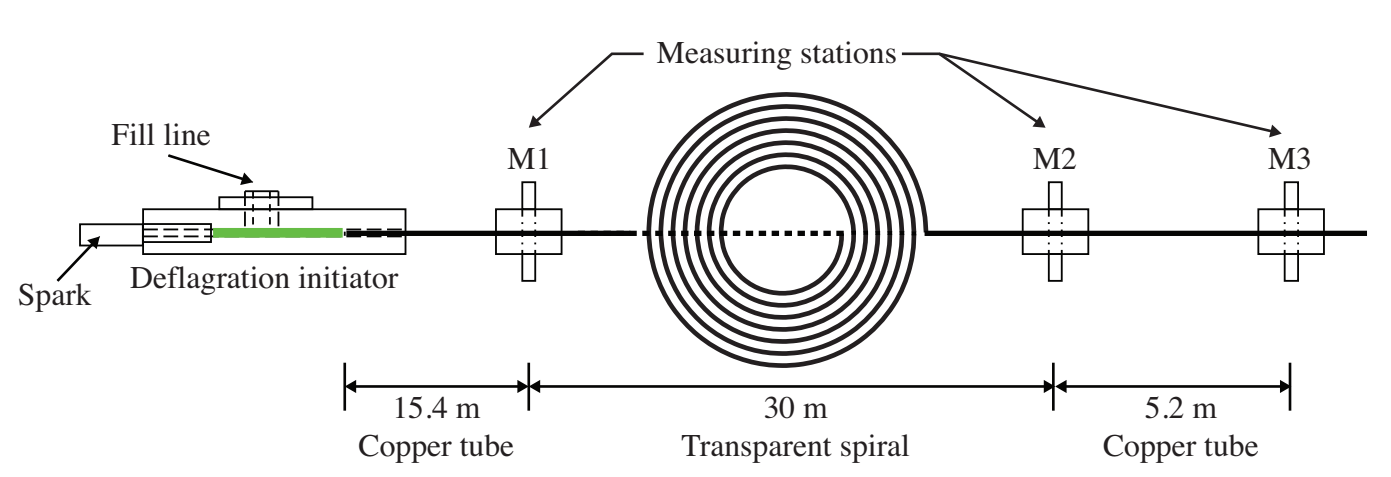

Figure 2: The experimental geometry.

was initiated with a $40 \mathrm{~mJ}$ spark. The resulting combustion wave then passed through a 15.4-m length of 4.8-mm inner diameter copper tubing to allow it to relax from the initiation event before reaching the first of three average velocity measurement stations. Each average velocity station simultaneously measured pressure, ionization, and luminescence, though only pressure data from the piezoelectric (PCB 113A24) transducers were used to determine average velocities in the present work. A section of transparent, polyurethane tubing was located downstream of the first measurement station. The tubing had an inner diameter of 4.1$\mathrm{mm}$, a 30-m-long observation section, and was coiled in a spiral configuration; the radius of the spiral ranged from 0.2 to $0.5 \mathrm{~m}$. The exit clear tubing exhausted into the second and third velocity measurement stations, which were separated by an additional 5.2-m length of copper tubing.

Prior to each experiment, the assembly was evacuated with a vacuum pump and then filled to the initial test pressure with stoichiometric propane-oxygen mixtures that were premixed in a separate mixing vessel. Initial pressures tested ranged from 6.5 to $20.0 \mathrm{kPa}$. Tests at $6.5 \mathrm{kPa}$ were unable to initiate sustained combustion, while all others transitioned to steady or galloping detonation.

The chemiluminescence associated with combustion in the polyurethane spiral was imaged with a high-speed framing camera (Vision Research Phantom V5) running at $12 \mathrm{kfps}(\Delta t=83.3 \mu$ s interframe time) with a resolution of $256 \mathrm{px}$ $\times 256 \mathrm{px}$ and an exposure time of $40.0 \mu \mathrm{s}$. Examples of the resulting images 
are shown in Fig. 3. As discussed in the subsequent sections, the images were processed to obtain a time series of the luminous wave front location and analyzed to obtain velocity statistics.

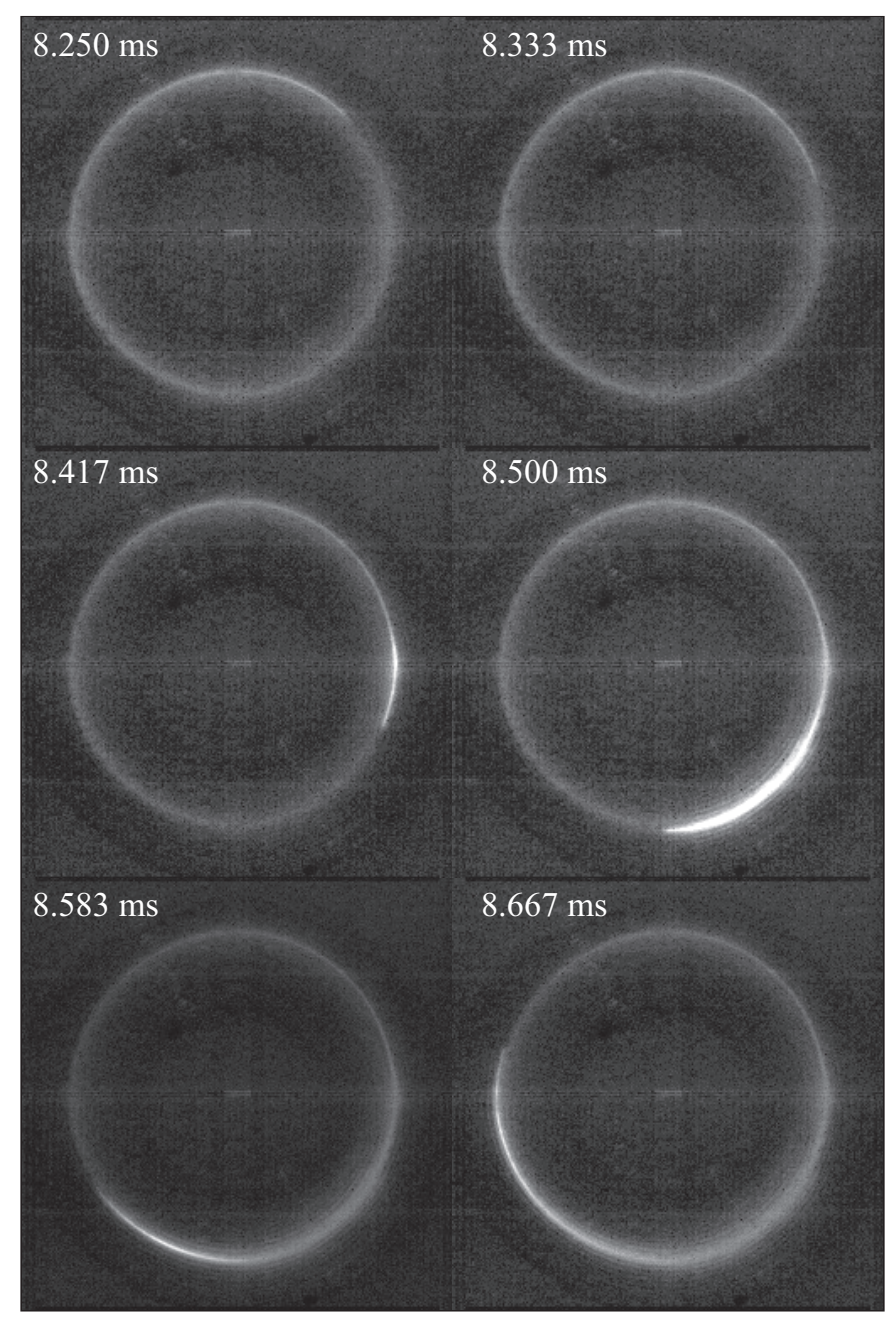

Figure 3: A series of images of chemiluminescence associated with the detonation propagation through the spiral geometry from test 198. The bright region in third frame is associated with a DDT event. The luminosity increase associated with the detonation relative to the preceding deflagration is apparent. The corresponding velocity time history is shown in Fig. A.28 using a common time base with these images. 


\section{Image Processing Procedure}

Comparison of the location of the luminous wave front in sequential images allowed determination of the wave velocity between each frame. These velocities were computed as follows. First, the image pixel coordinates $\left(x_{\mathrm{i}}, y_{\mathrm{i}}\right)$ associated with the leading edge of the combustion wave were identified, as shown for the example in Fig. 4. The combined result shows the wave progress through the spiral

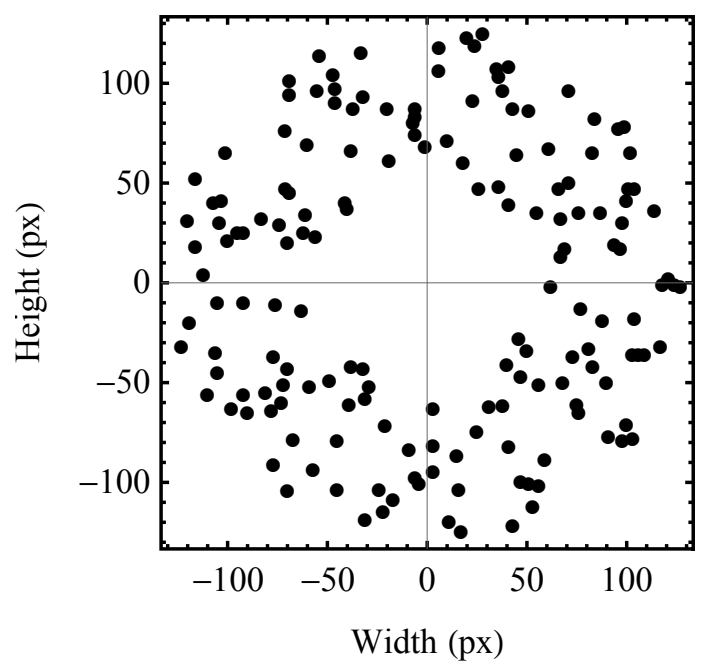

Figure 4: Wavehead CCD chip positions from each frame for test 195.

110

111

at each imaging timestep. The radius of each point $r_{\mathrm{i}}=\sqrt{\left(x_{\mathrm{i}}-x_{\mathrm{c}}\right)^{2}+\left(y_{\mathrm{i}}-y_{\mathrm{c}}\right)^{2}}$ was then calculated versus the image frame number as shown in Fig. 5. Some

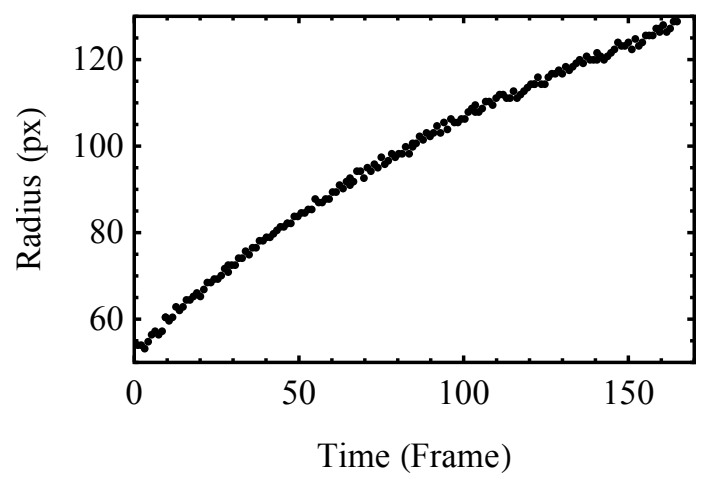

Figure 5: Wavehead radius versus time for test 195. 
$$
\text { (1) }
$$

small shift was present in the imaging setup from shot to shot that was typically on the order of $0-3$ pixels in both axes. In order to account for this shift, all data points from each test were used to solve for the common spiral center $\left(x_{\mathrm{c}}, y_{\mathrm{c}}\right)$ with subpixel resolution that yielded a smooth radius-versus-time curve. (Not accommodating this shift in image center would result in small oscillations in the inferred radius with time.) With the image center known, the angle of the leading edge of the luminous wave in the spiral was determined from $\phi_{\mathrm{i}}=\arctan \left(\frac{y_{\mathrm{i}}-y_{\mathrm{c}}}{x_{\mathrm{i}}-x_{\mathrm{c}}}\right)$; an example is shown in Fig. 6. With the radius and angle

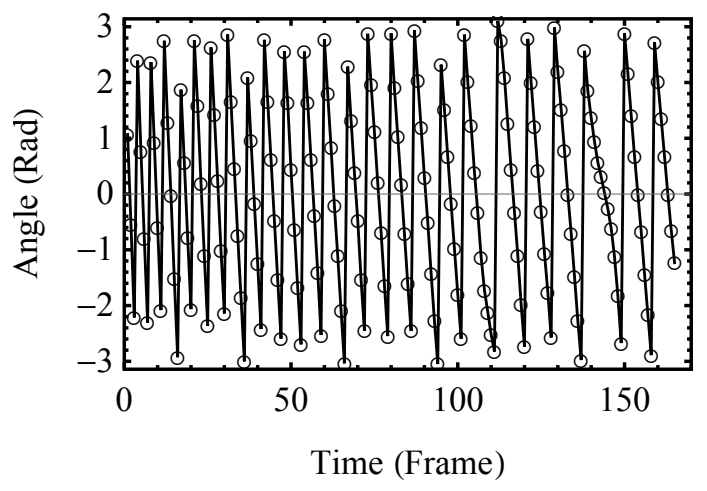

Figure 6: Spiral angle versus time for test 195.

120

evolutions in time known, the wave motion was then determined from the shifts across each frame $\Delta L_{\mathrm{i}}=\frac{1}{2}\left(r_{\mathrm{i}}+r_{\mathrm{i}-1}\right)\left(\phi_{\mathrm{i}}-\phi_{\mathrm{i}-1}\right)$. Normalization by the imaging timestep $\Delta t$ and multiplying by the calibration factor, $w=1.984 \mathrm{~mm} / \mathrm{px}$, gives the luminous front velocity for each timestep, $D_{\mathrm{i}}=w \Delta L_{\mathrm{i}} / \Delta t$, as shown in Fig. 7. A

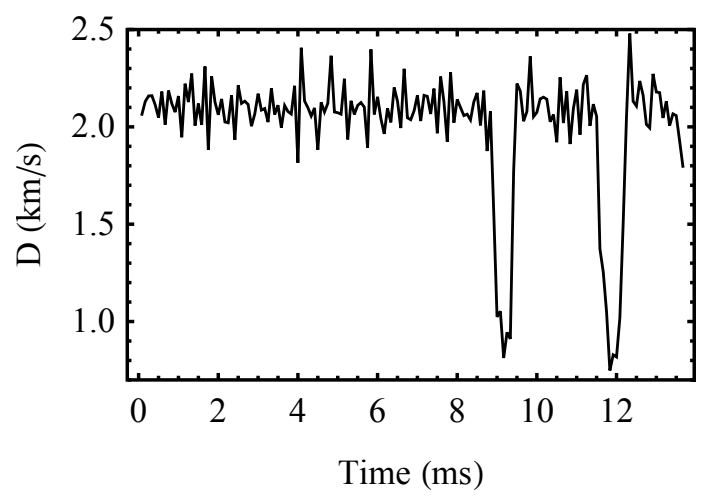

Figure 7: Detonation velocity versus time for test 195. 


\section{yie}

wavehead location uncertainty of 1 pixel (along the spiral) with the above analysis yields a velocity uncertainty of $\pm \Delta D_{\mathrm{i}} / D_{\mathrm{i}}=1 / \Delta L_{\mathrm{i}}$. This equation holds for all tests and yields the uncertainty magnitude shown in Fig. 8. We also note the pos-

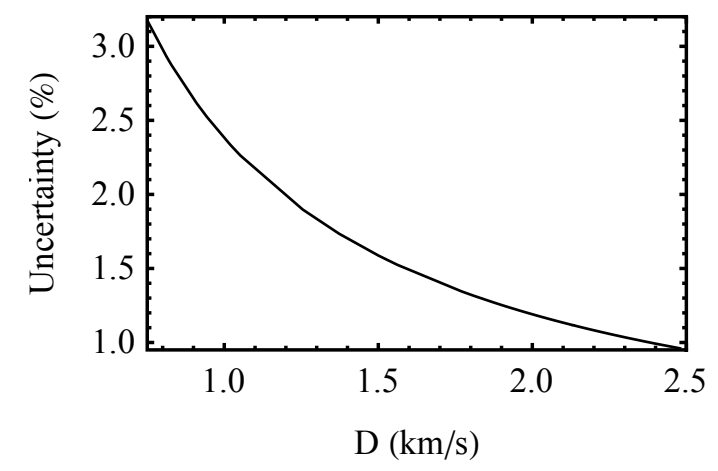

Figure 8: Uncertainty, in relative detonation velocity $D / D_{C J}$, associated with one pixel of error versus detonation velocity for all tests.

sibility of pixel identification error associated with varying chemiluminescence intensity versus wave speed and the finite image integration time (imaging blur) was likely \pm 3 pixels resulting in fluctuations with worst-case errors of up to $8 \%$ in $D / D_{\mathrm{CJ}}$. Our steady detonation velocity tests yield velocities of $0.95 \mathrm{D} / \mathrm{D}_{\mathrm{CJ}}$ with seemingly random fluctuations of $\pm 5 \%$, consistent with the above error analysis and average velocity measurements from the pressure transducers. Fluctuations of a similar character and magnitude are also observed in the time-resolved measurements of Lee et al. [2] and Haloua et al. [4] that were obtained with a completely different diagnostic (microwave interferometry). We conclude that the observed fluctuations are consistent with the combined physical and instrumental variation typical of this type of testing.

\section{Results}

\subsection{Average Velocities}

The average velocity results from the pressure measurement stations are shown in Fig. 1. The initial mixture pressure range of 7 to $20 \mathrm{kPa}$ yielded average detonation velocities between 0.57 and $0.94 D_{\mathrm{CJ}}$. In contrast, $c_{0} / D_{\mathrm{CJ}}$ is approximately 0.13 , where $c_{0}$ is the ambient sound speed of the mixture. Tests at $6.5 \mathrm{kPa}$ did not result in sustained propagation of a combustion wave. The figure also shows prior data from shorter length experiments with metal tubing at comparable diameters 

wave speed.

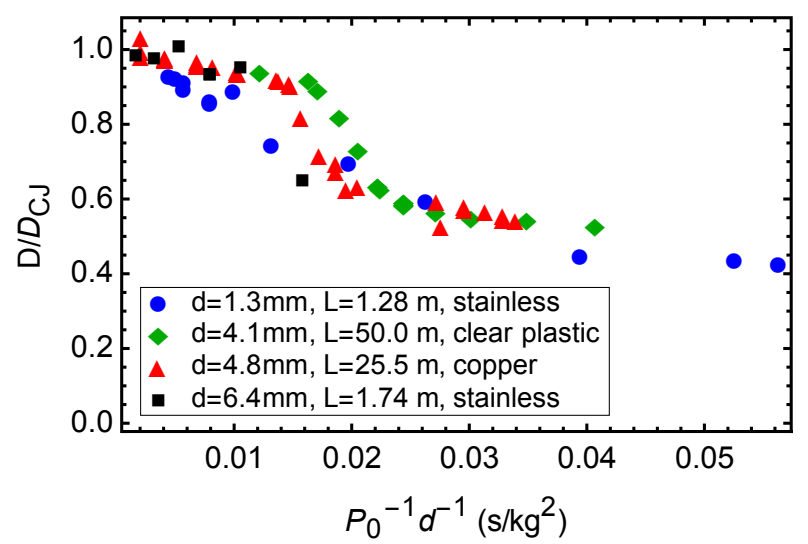

Figure 9: Average velocity data from Fig. 1 versus inverse binary scaling parameter $\left(1 / P_{0} d\right)$.

$[6,8,9]$ of $1.3 \mathrm{~mm}$ with straight tubing, $4.8 \mathrm{~mm}$ with spiral tubing, and $6.4 \mathrm{~mm}$ with straight tubing. The current data from the clear plastic tubing is seen to agree well with prior experiments at 4.1- and 6.4-mm. This agreement indicates that the detonation behavior in the plastic tubing is consistent with that in metal tubing. Thus, there is no evidence that the plastic tube wall is decomposing during testing for the conditions reported. Additionally, results of the experiments in the spirals compare well with those from the straight $6.4-\mathrm{mm}$ diameter tubing, indicating that the spiral geometry does not appear to be influencing the wave dynamics.

In Fig. 9, the data have been rescaled as a function of $1 / P_{0} d$ in anticipation of the importance of binary collisions in the high temperature chemistry. For a given mixture composition, tests with similar values of $P_{0} d$ will have similar ratios of chemical reaction and physical length scales if binary collisions dominate the reaction processes [15]. This is known as binary scaling and the reasonable collapse of the data in these coordinates indicates that the ratio of reaction length scales to tube diameter is a key parameter in a model of the effect of tube size on

\subsection{Unsteady Velocity Measurements and Mode Probability}

The velocity data derived from the framing camera analysis provided wave velocity measurements with high temporal resolution, allowing visualization of any unsteady wave motion. The velocity histories from tests at different pressures are summarized in Figs. A.23-A.38, with the results shown in order of decreasing pressure. The left image for each test shows the measured velocity record versus time. The right image shows a histogram representing the relative frequency 
or experimental probability of each velocity present in the test with the velocity binned in $0.05 D_{\mathrm{CJ}}$ bin widths. The results are summarized in Table 1, which also contains cell size $\lambda$ and effective activation energy $\theta$ data. Both $\lambda$ and $\theta$ are computed from fits. Parameter $\lambda$ is fit to data in Kaneshige and Shepherd [14] to yield $\lambda=186.5 P_{0}^{-1.173}$ with $P_{0}$ in $\mathrm{kPa}$ and $\lambda$ in mm. Parameter $\theta$ is fit to data from Schultz and Shepherd [12] to yield $\theta=12.77 P_{0}^{-0.0636}$ with $P_{0}$ in kPa. Previously, multiple criteria for the onset of spin detonation have been proposed with $\lambda / d=$ 2 or $\pi$ [16]; the present experimental series exhibits galloping onset at some point between $\lambda / d=1.9$ and 2.5 .

Table 1: Test data summary.

\begin{tabular}{|cccccccc|}
\hline $\begin{array}{c}\text { Test } \\
\text { No. }\end{array}$ & $\begin{array}{c}P_{0} \\
(\mathrm{kPa})\end{array}$ & $\begin{array}{c}D_{\mathrm{CJ}} \\
(\mathrm{mm} / \mu \mathrm{s})\end{array}$ & $\mathrm{D} / D_{\mathrm{CJ}}$ & $\begin{array}{c}\text { Observed } \\
\text { Mode }\end{array}$ & $\begin{array}{c}\lambda \\
(\mathrm{mm})\end{array}$ & $\lambda / d$ & $\theta$ \\
\hline 190 & 20.1 & 2.287 & 0.939 & Steady & 5.52 & 1.35 & 10.55 \\
189 & 19.9 & 2.287 & 0.934 & Steady & 5.59 & 1.36 & 10.56 \\
191 & 15.1 & 2.274 & 0.893 & Stuttering & 7.72 & 1.88 & 10.75 \\
195 & 15.0 & 2.274 & 0.876 & Stuttering & 7.78 & 1.90 & 10.75 \\
192 & 12.0 & 2.264 & 0.658 & Galloping & 10.1 & 2.47 & 10.90 \\
198 & 12.0 & 2.264 & 0.670 & Galloping & 10.1 & 2.47 & 10.90 \\
194 & 10.1 & 2.256 & 0.610 & Galloping & 12.4 & 3.02 & 11.03 \\
193 & 10.0 & 2.256 & 0.605 & Galloping & 12.5 & 3.05 & 11.03 \\
202 & 9.0 & 2.252 & 0.580 & Galloping & 14.2 & 3.46 & 11.11 \\
203 & 9.0 & 2.252 & 0.584 & Galloping & 14.2 & 3.46 & 11.11 \\
196 & 8.0 & 2.247 & 0.581 & Galloping & 16.3 & 3.97 & 11.19 \\
197 & 8.0 & 2.247 & 0.568 & Galloping & 16.3 & 3.97 & 11.19 \\
199 & 7.6 & 2.244 & 0.572 & Galloping & 17.3 & 4.21 & 11.23 \\
204 & 7.6 & 2.244 & 0.569 & Galloping & 17.3 & 4.21 & 11.23 \\
200 & 7.1 & 2.241 & 0.567 & Galloping & 18.7 & 4.56 & 11.27 \\
201 & 7.0 & 2.241 & 0.566 & Galloping & 19.0 & 4.64 & 11.29 \\
\hline
\end{tabular}

A clear trend is observed from the histograms with a systematic change in propagation characteristics with decreasing initial pressure (increasing values of $\lambda / d$, see Table 1). In the steady and stuttering detonation regime above $15.0 \mathrm{kPa}$, the dominant velocity mode is $0.95 D_{\mathrm{CJ}}$. In the galloping regime for pressures less than $15 \mathrm{kPa}$, bimodal behavior occurs, with dominant modes having a luminous front velocity of $0.4 D_{\mathrm{CJ}}$ and $0.95 D_{\mathrm{CJ}}$. These two dominant modes are consistent throughout the galloping regime. Additionally, a broader distribution of velocities occurs in the galloping region than in the steady detonation regime. As $P_{0}$ decreases, the probability of the $0.95 D_{\mathrm{CJ}}$ mode decreases until, at $8 \mathrm{kPa}$, it is barely significant as a peak. Compiling the histogram data into only two bins 

binned in $0.1 D_{\mathrm{CJ}}$ increments. The PDF clearly shows a single peak near $0.95 D_{\mathrm{CJ}}$
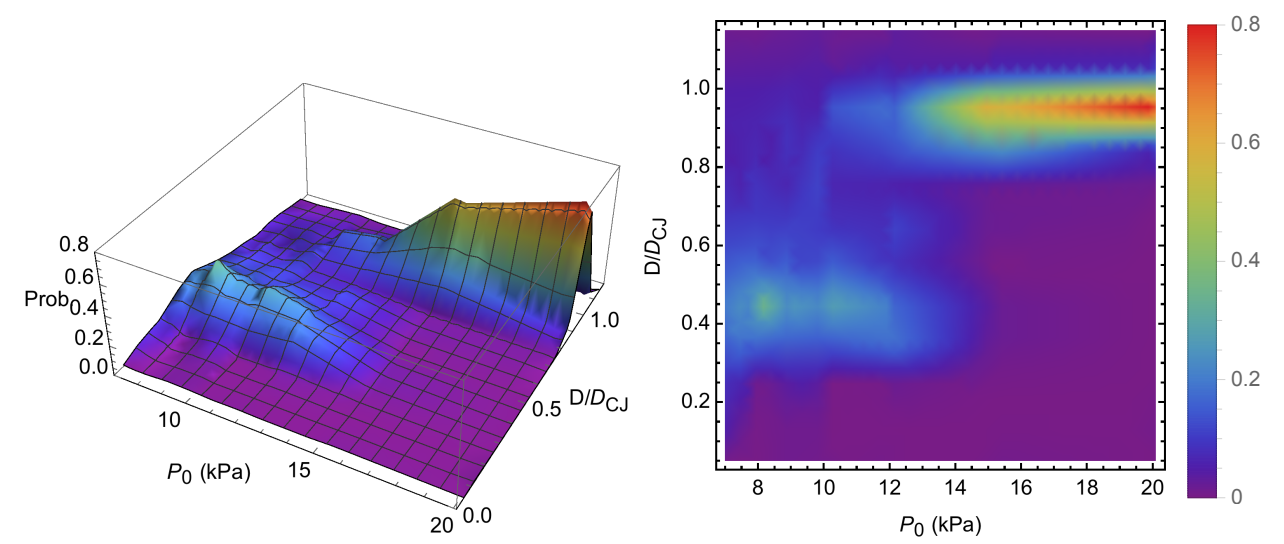

Figure 10: Probability distribution function of the velocity modes with initial mixture pressure.

centered on $0.4 D_{\mathrm{CJ}}$ and $0.95 D_{\mathrm{CJ}}$, the ratio of low-to-high speed velocity occurrences is 1:99 for $19.9 \mathrm{kPa}$ (Fig. A.24), $56: 44$ for $12.0 \mathrm{kPa}$ (Figure A.28), 67:33 for $10.1 \mathrm{kPa}$ (Figure A.29), and 73:27 for $8.0 \mathrm{kPa}$ (Figure A.33).

The velocity-time history reflects this behavior. Tests at 20.1 and $19.1 \mathrm{kPa}$ (Figs A.23 and A.24) show steady detonation propagation near $0.95 D_{\mathrm{CJ}}$ with small and intermittent velocity perturbations. The tests at $15 \mathrm{kPa}$ (Figs. A.25 and A.26) are characteristic of stuttering detonation (as defined by Lee et al. [2]), where the detonation wave briefly fails and drops down to $0.4 D_{\text {CJ }}$ before quickly reinitiating. In the galloping detonation regime at $12 \mathrm{kPa}$, the wave appears to spend approximately equal amounts of time at both $0.40 D_{\mathrm{CJ}}$ and $0.95 D_{\mathrm{CJ}}$, with rapid transitions in between each mode associated with detonation failure and reignition. As the initial pressure is decreased to even lower values of 8-12 $\mathrm{kPa}$, (Figs. A.27-A.33), less time is spent near $0.95 D_{\mathrm{CJ}}$, a continuous decrease in the average wave velocity is observed which is consistent with lower-resolution measurements (Fig. 1). At $8 \mathrm{kPa}$ (Fig. A.33), the wave consistently and immediately fails upon reaching $0.95 D_{\mathrm{CJ}}$.

Figure 10 compiles these results into a three-dimensional surface representing the probability distribution function (PDF) for the observed velocity modes versus initial mixture pressure. The PDF was generated by first-order (linear) interpolation of the individual test histogram data (Figs. A.23-A.38) in both the $P_{0}$ and $D / D_{\mathrm{CJ}}$ dimensions with relative frequencies computed from the $D / D_{\mathrm{CJ}}$ data rebirned in $0.1 D_{\mathrm{CJ}}$ increments. The PDF clearly shows a single peak near $0.95 \mathrm{D}$

from 20 to $15 \mathrm{kPa}$. At pressures less than $15 \mathrm{kPa}$, a bimodal distribution exists with peaks at both $0.95 D_{\mathrm{CJ}}$ and $0.4 D_{\mathrm{CJ}}$, which is characteristic of an oscilla- 


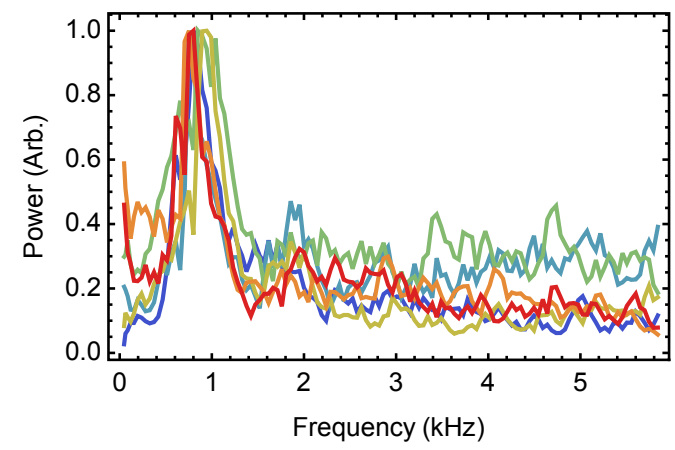
to detonation [16]. cycles. these concerns.

tory signal. For $P_{0}>12.5 \mathrm{kPa}$, the $0.95 D_{\mathrm{CJ}}$ velocity regime is more probable, consistent with ordinary detonation wave propagation. For $P_{0}<12 \mathrm{kPa}$, the 0.4 $D_{\mathrm{CJ}}$ velocity regime is most probable, consistent with a high speed deflagration or a shock wave trailed by a decoupled fast flame, which is generally the highest flame velocity observed before a local explosion transitions the deflagration mode

These results are consistent with prior work, particularly that of Lee et al. [2], which qualitatively demonstrated the evolution of steady detonation waves into the stuttering and galloping regimes for various mixtures and applied a similar histogram analysis. The present study, however, demonstrates the full velocity mode evolution for a single unstable mixture with a probability distribution function and shows that the galloping mode is regular and repeatable for up to 20

\subsection{Frequency Analysis of the Galloping Regime}

In this work, the combination of small tubing diameter and extremely long observation lengths $(L / d>7,300)$ were sufficient to allow measurement of many galloping cycles (up to 20). This is in contrast to earlier efforts that generally were able to only observe $1-5$ galloping cycles, leaving open the questions of (1) if the measured detonation failure was true detonation failure or just an increase in the galloping cycle length and (2) if there was any dependence of the tube length or ignition mechanism on the measured results. The larger number of cycles observed in this study allows for the application of statistical techniques to address

Figure 11: The galloping frequency vs. pressure. Left plot shows FFT data for 8.0 (blue), 9.0 (teal), 10.0 (green), 10.1 (yellow), 12.0 (orange and red) $\mathrm{kPa}$. Right plot shows power spectrum (blue is low and red is high) versus frequency and initial mixture pressure. 
Figure 11 shows the measured galloping frequency versus initial mixture pressure. Galloping frequencies were evaluated by applying a Fast Fourier Transform (FFT) to the velocity-time history from each test. The left portion of Fig. 11 shows an overlay of the individual results from all tests in the galloping regime; a single dominant frequency is observed in each test that is between 0.8 and $1.0 \mathrm{kHz}$. The right component of Fig. 11 interpolates the FFT results onto a surface plot of frequency versus initial pressure. The dominant frequency does not significantly vary with mixture pressure throughout the entire observed galloping regime (7$12 \mathrm{kPa}$ ). In the steady detonation regime above $15 \mathrm{kPa}$, no strong frequencies are observed. In the transitional stuttering regime, at $15 \mathrm{kPa}$, frequencies near 450 $\mathrm{Hz}$ and below $100 \mathrm{~Hz}$ are detected. The frequency below $100 \mathrm{~Hz}$ may be significant in the range between 12 to $15 \mathrm{kPa}$, though the temporal record length in the present study is not adequate to resolve such low values and the low frequency oscillations did not persist below $12 \mathrm{kPa}$. The observation of a nearly universal $0.8-1.0 \mathrm{kHz}$ frequency band associated with galloping is striking, given that analysis of the velocity modes demonstrated a monotonic decrease in the probability of the $0.95 D_{\mathrm{CJ}}$ velocity mode with decreasing pressure.

\subsection{Quantitative Breakdown of the Galloping Regime}

Analysis of the individual components or phases of the oscillation cycle of the galloping regime is revealing and may be useful in developing physical models of the processes that contribute to this behavior. The velocity histories of Section 4.2 indicate that galloping regime is composed of four possible components or phases: (1) wave dwell near a $0.4 D_{\text {CJ }}$ phase, (2) wave acceleration (likely through DDT) to a $0.95 D_{\text {CJ }}$ phase, (3) wave dwell near this $0.95 D_{\text {CJ }}$ phase, and (4) wave deceleration (likely through detonation failure and subsequent shock wave attenuation) to the $0.4 D_{\mathrm{CJ}}$ phase.

Figure 12 overlays the low-velocity wave dwell and combustion wave acceleration profiles from a test at $8 \mathrm{kPa}$ (test 196) in black and a test at $12 \mathrm{kPa}$ (test 198) in red. Scatter or fluctuations are present, but the timing and velocity magnitude associated with the DDT phenomena are seen to be invariant of the initial mixture pressure. Combustion wave profiles associated with the wave deceleration and subsequent low-velocity dwell phases are overlaid in Fig. 13 for the same two tests. The detonation failure trajectories demonstrate a similar insensitivity to initial pressure. In fact, in addition to being independent of pressure, both the DDT and failure trajectories appear to occupy a similar period of approximately 7.5 imaging frames. The period associated with DDT that was immediately followed by failure would, thus, be 15 imaging frames or $1.25 \mathrm{~ms}$. This period corresponds 


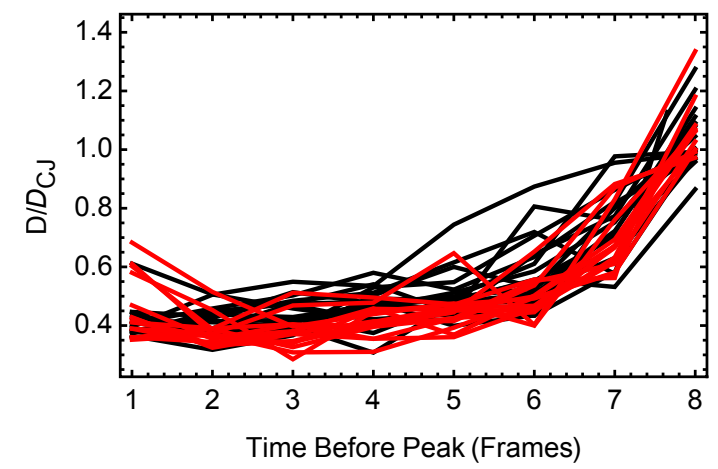

Figure 12: Wave acceleration profiles from galloping detonation at $8 \mathrm{kPa}$ (test 196) in black and $12 \mathrm{kPa}$ (test 198) in red.

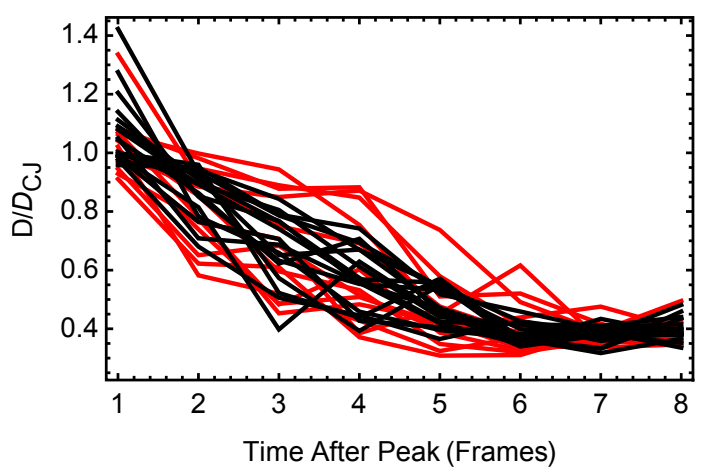

Figure 13: Wave deceleration profiles from galloping detonation at $8 \mathrm{kPa}$ (test 196) in black and $12 \mathrm{kPa}$ (test 198) in red.

to a frequency of $0.8 \mathrm{kHz}$, which is close to that yielded by the FFT analysis. Thus, wave acceleration and deceleration or mode switching process correlates well with the measured galloping frequency.

The amount of time the galloping wave spends at both the high- and lowvelocity phases was also evaluated. This was done by manually selecting portions of the wavefront in each phase. High-velocity dwell times consisted of the time between the peak cycle velocity and the onset of the steep gradient indicating decay to the low-velocity phase, in integer frame numbers. Low-velocity dwell times consisted of the time between the termination of this steep decay gradient and the onset of the subsequent steep acceleration gradient associated with DDT. During the high-velocity and low-velocity dwell times, all wave speeds were generally above $0.8 D_{\mathrm{CJ}}$ or below $0.6 D_{\mathrm{CJ}}$ respectively. An example of these selected 
regions for test 198 is shown in Fig. 14. Performing this analysis across all tests

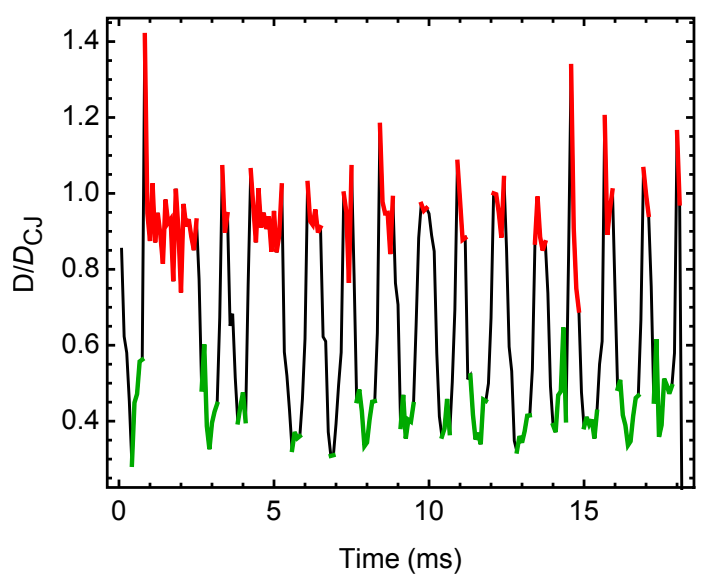

Figure 14: Portions of the wavefront in the high velocity (red) and low velocity (green) phase for test 198 .

allows determination of the mean dwell time per cycle that the wave spends in both the high velocity and low velocity phases and how these dwell times vary with initial mixture pressure. Figure 15 shows these data with mean high velocity times in red (squares) and mean low velocity times in green (circles). Empirical curves are also fit to the data to capture the trends in a concise form. The green and red curves are exponential functions of the form $\tau=\exp \left(A+B P^{C}\right)$. The fitted parameters for the green curve are $A=-0.849, B=-1.06 \times 10^{-6}$, $C=4.89$ with $P$ in $\mathrm{kPa}$ and $\tau$ in ms. For the red curve, they are $A=-2.89$, $B=1.841 \times 10^{-4}, C=3.74$ with identical units. Dwell time spent in the low velocity phase is seen to be fairly insensitive of pressure. In contrast, the dwell time of the high velocity mode is strongly dependent on pressure. Attempting to fit the dwell time data with a power law $\tau=B P^{C}$ achieves a worse fit. With the power law form, the green curve fit parameters are $B=0.915 \mathrm{~ms}$ and $C=-0.392$ with $P$ in $\mathrm{kPa}$. An acceptable fit for the red curve is only achieved by discarding the $15 \mathrm{kPa}$ experimental points from the fit; the remaining data then fits well to $B=4.95 \times 10^{-5} \mathrm{~ms}$ and $C=3.59$ with $P$ in $\mathrm{kPa}$.

Figure 16 shows the mean amount of time (left image) and percentage (right image) spent in each phase of the galloping cycle as a function of initial mixture pressure. Again, only the dwell time at the high-velocity phase appears strongly pressure dependent, increasing to almost $90 \%$ of the cycle time at $15 \mathrm{kPa}$. Below $10 \mathrm{kPa}$, the dwell time spent in the high-velocity phase becomes very short 


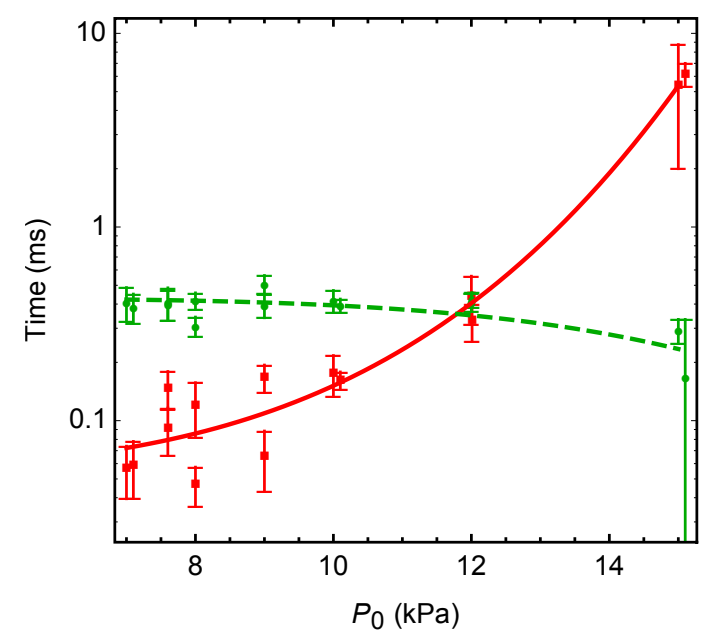

Figure 15: Mean wave dwell times in the high velocity (squares, red solid curve) and low velocity (circles, green dashed curve) phases versus initial mixture pressure. The error bars represent standard error computed from the variance associated with the dwell times.
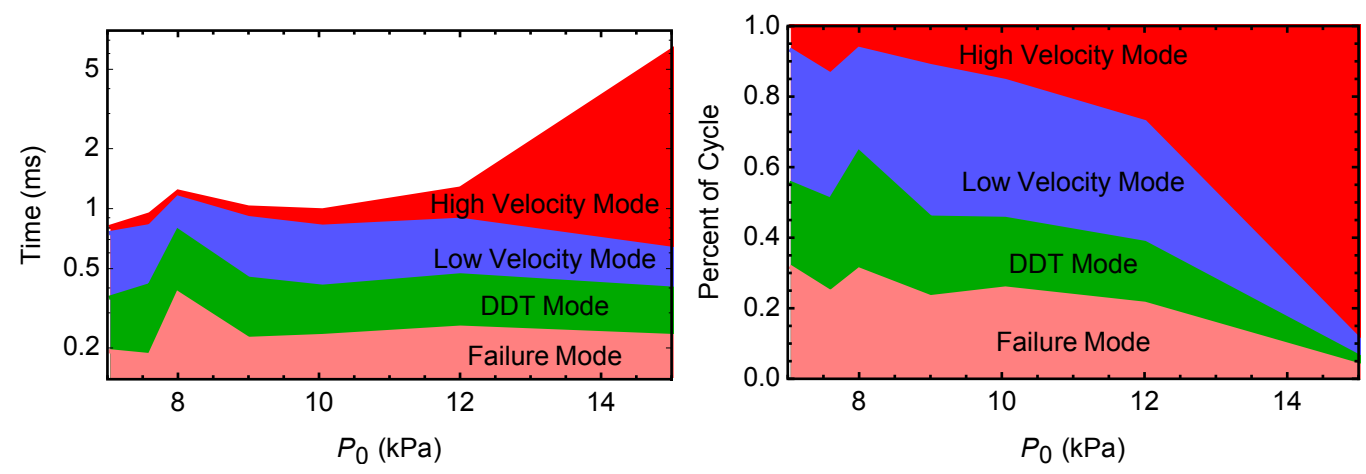

Figure 16: Time (left) and percentage (right) of each phase of the galloping cycle versus initial mixture pressure.

(Fig. 16) and the scatter between tests at repeat conditions increases (Fig. 15). Qualitative inspection of Figs. A.23-A.38 also indicates that, below this limit, the velocity-time behavior no longer exhibits a plateau at the high-velocity mode. We interpret the combination of these two observations to indicate repeated onset of the DDT mechanism followed by its consistent failure before the process achieves detonation. There is also a local increase in the period of the DDT and failure phases at $8 \mathrm{kPa}$; the reason for this is currently not known. The total galloping cycle time is slightly above $1 \mathrm{~ms}$ for initial pressures of $8-12 \mathrm{kPa}$, consistent with 
the FFT peak between 0.8 and $1.0 \mathrm{kHz}$ in this regime.

\subsection{Phase Plane and Bifurcation Analysis}

Instabilities associated with one-dimensional (1D) detonation are manifested as longitudinal pulsations of the leading shock wave (see for example Zhang et al. [17], Kasimov and Stewart [18]) which are superficially similar to the oscillations of the luminous front observed in the present study. For simplified models of reaction rate and thermochemistry, results of $1 \mathrm{D}$ numerical simulations have been analyzed by using methods from nonlinear dynamical systems by $\mathrm{Ng}$ et al. [19], Henrick et al. [20], Abderrahmane et al. [21]. With increasing activation energy, a sequence of dynamical states is observed beginning with stable waves, onset of instability, limit cycles with a single period, then multiple periods, and ultimately aperiodic behavior characteristic of deterministic chaos. This sequence of events is also valid when transport effects (viscous and heat conduction) are included in strictly 1D simulations [22] and evidence of this is also observed [23] when using channel flow approximations to model the effects of friction through a wall function. Multidimensional detonations with transport effects are much more challenging to accurately simulate with realistic channel boundary conditions so that only modest progress [24] has been made in applying dynamical systems analyses to these cases.

Motivated by the application of dynamical systems methods to numerical simulations, we have analyzed the experimental velocity-time series using phase plane diagrams and bifurcation graphs. In the present study, the effective activation energy is not a strong function of pressure so that initial pressure itself may be a more sensible choice of a control parameter although other choices such as $\lambda / d$ are equally valid.

Figures 17-21 show the oscillation modes present for selected tests in order of decreasing pressure, which is equivalent to increasing values of $d / \Delta$ or $d / \lambda$. The left images for each test show the measured velocity-time trace (in black), as discussed in Section 4.2 along with a filtered trace (in red) with high frequency noise removed. The right image visualizes the limit cycles of each filtered trace with a plot of front acceleration versus velocity. The filtered trace was fitted with a fifth-order interpolation function (shown in red), which was differentiated in order to generate the accelerations $\dot{D}$ used as the ordinate in the phase diagrams. All phase plane images are at the same scale and plot normalized acceleration $\dot{D} / D_{\mathrm{CJ}}$ versus normalized luminous front speed $D / D_{\mathrm{CJ}}$.

In the steady detonation regime (Fig. 17), small oscillations are observed around $0.95 D_{\mathrm{CJ}}$ that approximate a single orbit consistent with a single period 

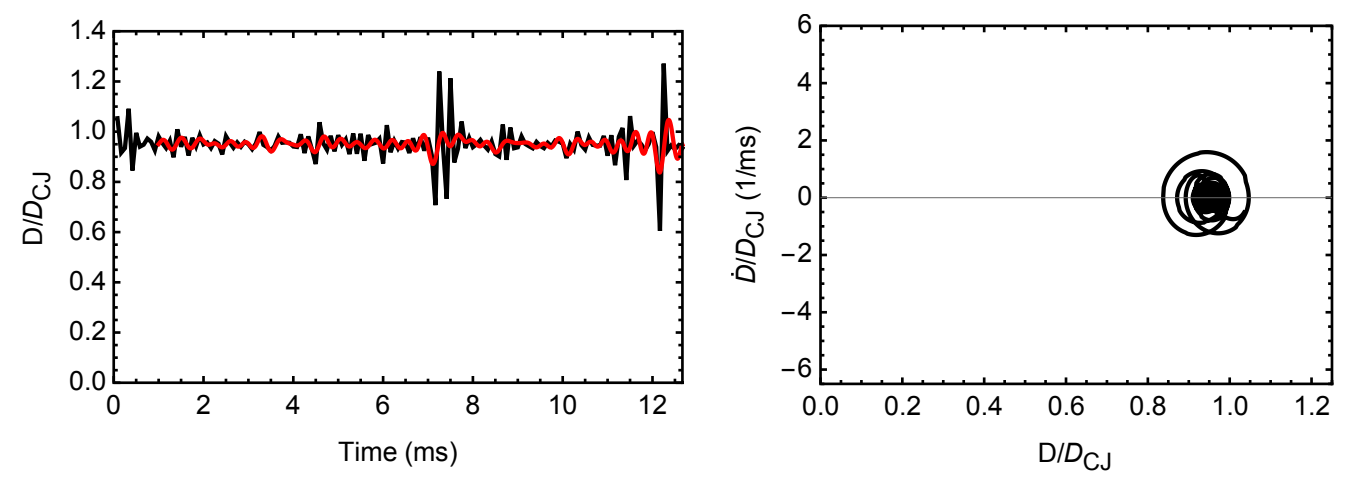

Figure 17: Velocity-time (left) and limit cycle (right) plots for test 189 at $19.9 \mathrm{kPa}$.
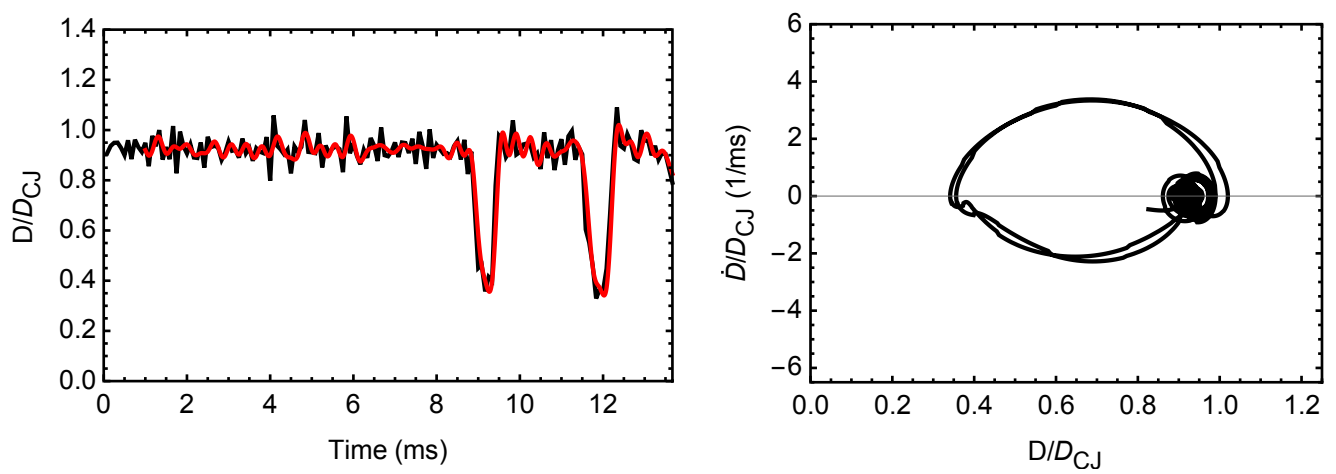

Figure 18: Velocity-time (left) and limit cycle (right) plots for test 195 at $15.0 \mathrm{kPa}$.
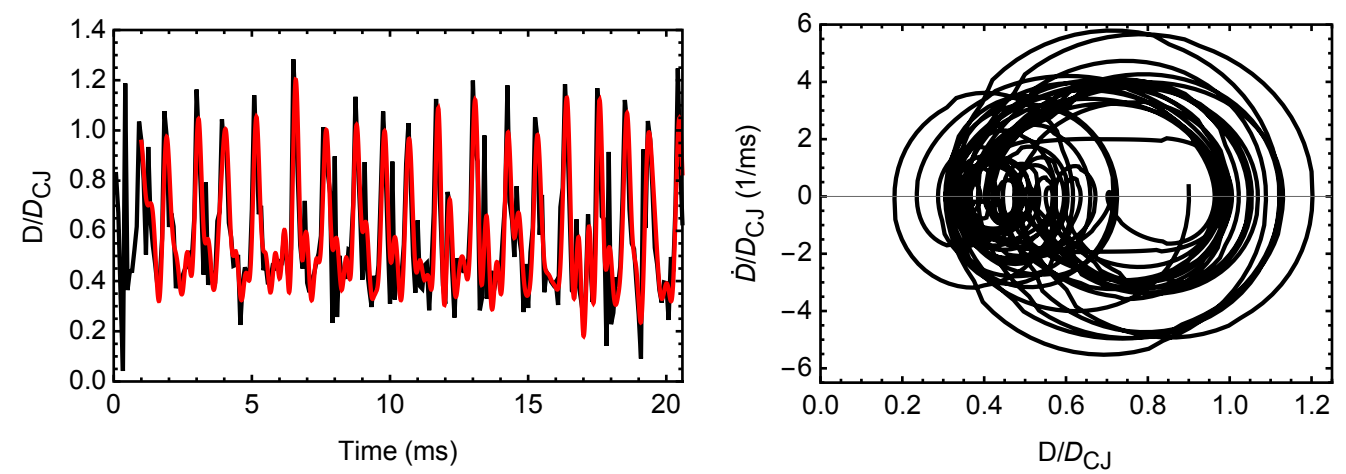

Figure 19: Velocity-time (left) and limit cycle (right) plots for test 203 at $9.0 \mathrm{kPa}$.

limit cycle. A period 2 limit cycle then appears to develop as the pressure is decreased into the stuttering regime (Fig. 18). In the above two cases, the limit 

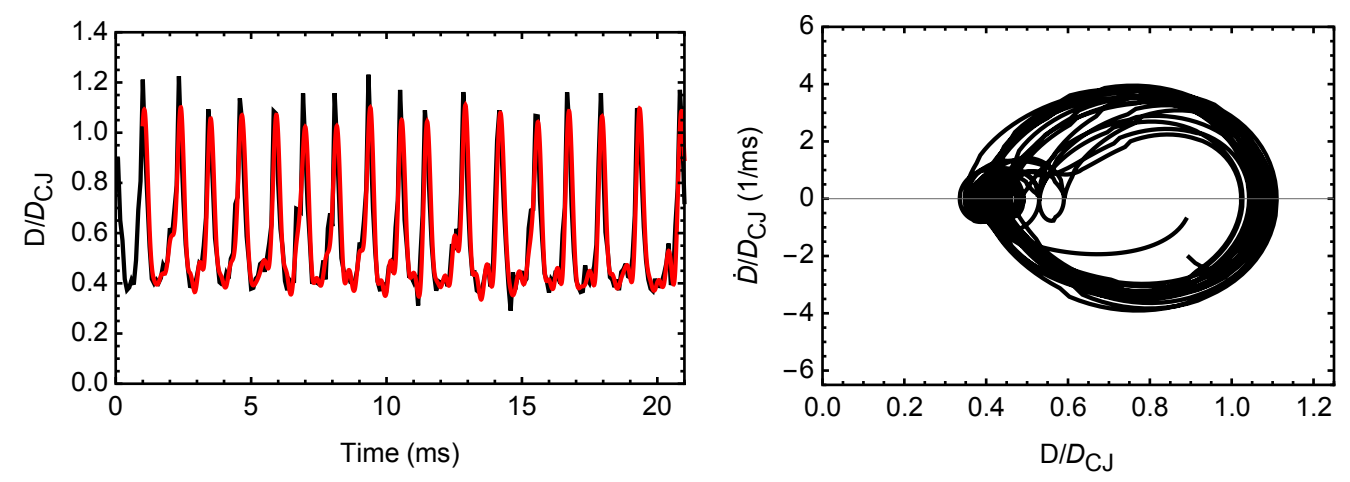

Figure 20: Velocity-time (left) and limit cycle (right) plots for test 197 at $8.0 \mathrm{kPa}$.
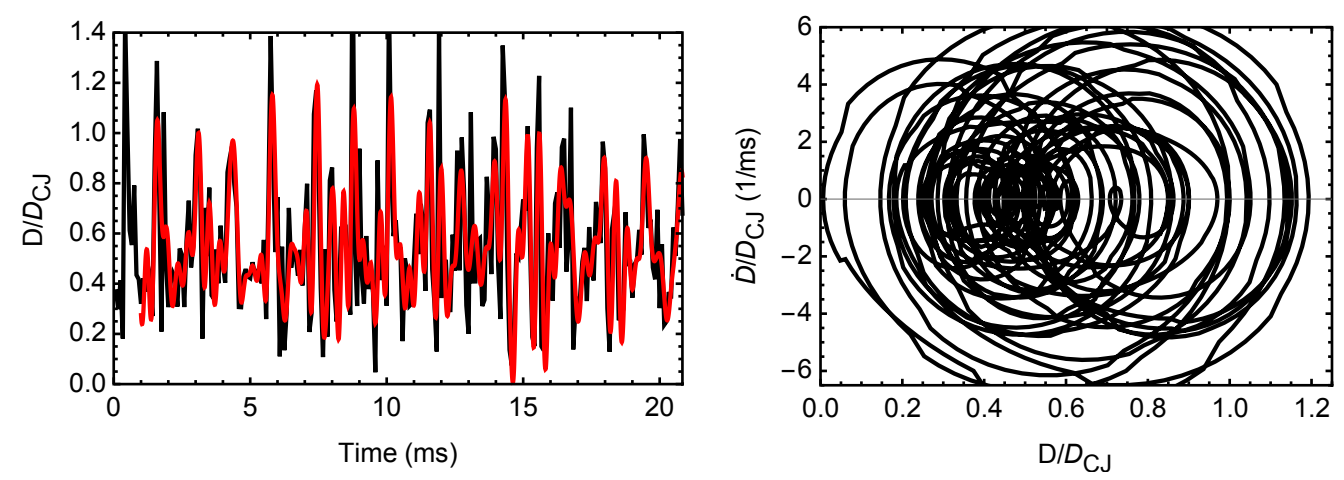

Figure 21: Velocity-time (left) and limit cycle (right) plots for test 201 at $7.0 \mathrm{kPa}$.

cycles appear to be relatively stable, within the experimental uncertainty. As the pressure continues to decrease to $9 \mathrm{kPa}$ (Fig. 19), the limit cycle becomes more complex and less stable, either exhibiting an increased number of periods or aperiodic behavior. It is difficult to tell given the limitations of our experiments and analysis techniques; particularly the finite temporal resolution and the inherently noisy nature of numerical differentiation. Periodic behavior re-appears at $8 \mathrm{kPa}$ (Fig. 20) and appears increasingly aperiodic as the pressure is reduced to $7.0 \mathrm{kPa}$ (Fig. 21).

Bifurcation diagrams are another technique from dynamical systems analysis that can be used to visualize the number of periods present in limit cycle in a more concise manner than the above phase diagrams. To construct these diagrams, the local maxima of the amplitudes of a measure of oscillation (leading shock pressure or velocity) are plotted against the control parameter that determines the system behavior, usually activation energy in the case of 1D detonation modeling. For 1D 
detonation stability studies $[19,20]$, period doubling is incrementally observed with increasing activation energy, from one-, to two-, to four-, to eight-, to 16period oscillations before the wave motion appears to become aperiodic. Further increase in the activation energy will often result in the resumption of periodic motion for a small range of activation energy, before the onset of a second region of aperiodic flow. More detailed examination [21] of the transition to aperiodic behavior demonstrates that this has the characteristics of deterministic chaos.

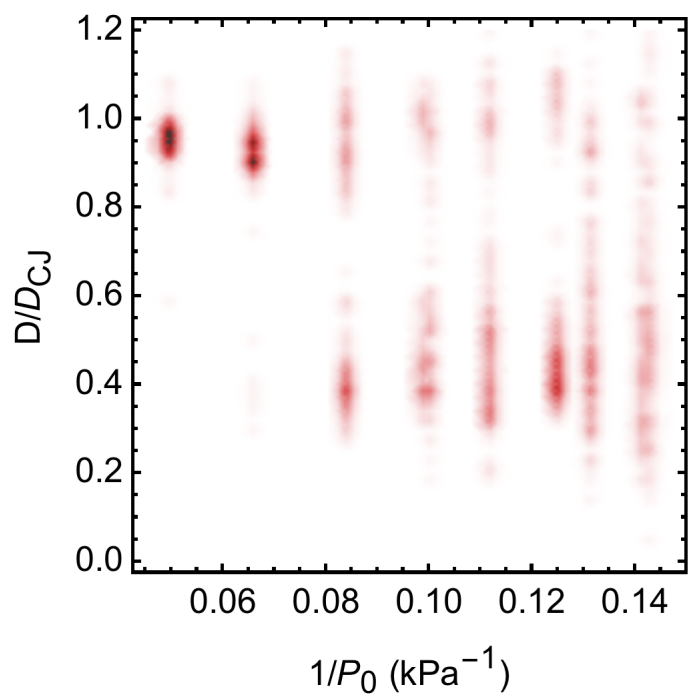

Figure 22: Local maxima and minima of velocity as a function of inverse initial pressure. Whiteto-black (with red intermediate coloring) indicates increasing probability.

The present wave velocity oscillations are more irregular than in the case of the numerical simulations and also contain experimental noise, making identification of an integer number of periods and characterization of the aperiodic motion more challenging. Despite these issues, we have made a first effort to create bifurcation graphs from our experimental data. Figure 22 presents our experimental bifurcation diagram in the form of a density plot, showing local maxima and minima in $D$ as calculated from the filtered velocity-time traces versus inverse initial mixture pressure. The number of periods is seen to increase and possibly double as $P_{0}$ decreases from 20 to $15 \mathrm{kPa}\left(0.050\right.$ to $\left.0.067 \mathrm{kPa}^{-1}\right)$ and possibly double again with a further decrease from 15 to $12 \mathrm{kPa}\left(0.067\right.$ to $\left.0.083 \mathrm{kPa}^{-1}\right)$. After that, the density distribution of data is more diffuse and consistent with aperiodic flow for pressures of 10 and $9 \mathrm{kPa}\left(0.100\right.$ to $\left.0.111 \mathrm{kPa}^{-1}\right)$. At $P_{0}=8 \mathrm{kPa}(0.125$ $\left.\mathrm{kPa}^{-1}\right)$, the data distribution becomes less diffuse, with two broadly defined peaks, 
before again becoming more diffuse as the pressure is further decreased below 8 $\mathrm{kPa}$. This trend is reminiscent of the brief onset of periodic flow in between aperiodic regions of the bifurcation diagrams constructed from numerical simulations $[19,20]$ of $1 \mathrm{D}$ detonations.

Our preceding analysis and interpretation draws heavily from prior studies on 1D numerical detonation stability. However, the multi-dimensional flow, losses, and real chemistry present in the experiments may introduce new dynamical behavior not previously observed in the 1D simulations. The experimental scatter and our smoothing approach may have masked these features from our identification. In this case, further experimental studies with increased resolution or numerical approaches with more complex losses and chemistry will be necessary to further explore these dynamics. Additionally, as noted, our study tracks the velocity of the front luminosity, in contrast to prior numerical studies that traditionally report lead shock velocity. As these two flow features repeatably couple and decouple in the galloping flow, their dynamics may have differences.

\section{Discussion}

The results of the present study are broadly consistent with both the recent experiments mentioned in the introduction to this paper as well as older studies (see the references in Tsuboi et al. [25] as well as in Ul'yanitskii [26]). In the present study, only the speed of the luminous front is recorded and it is not possible to distinguish between the speed of the leading shock wave and the trailing reaction zone that is the origin of the luminosity, as was carried out in some previous studies. However, our continuous recording technique and the long spiral test section enable much greater recording time of the luminous front speed than previous efforts, which had limited point measurements of arrival times and much shorter test sections.

The most striking result of the present study is the dependence of the wave speed distribution on initial pressure. The effect of initial pressure or mixture composition on the combustion wave behavior in small channels is conventionally explained in terms of the competition between friction associated with viscosity (molecular transport of momentum) and the pressure dependence of the chemical reaction rate in the gas behind the leading shock front. The effect of friction can be conceptualized from two points of view: (1) Stream tube expansion due to the boundary layer displacement effect. One of the first studies was by Fay [27], for a more recent examination of this issue, see Sow et al. [23]; (2) the net loss of momentum from the flow as reflected in the change in the 
mean velocity averaged across the channel or tube cross section, as modeled for example by Aksamentov et al. [28]. A thermal boundary layer is also present, representing the analogous competition between heat losses from the gas to the tube wall and the temperature dependence of the chemical reaction rate in the gas behind shock. Recent advances in computational capability have enabled direct numerical simulation of detonation propagation in a narrow channel with the twodimensional Navier-Stokes equations with viscous (but no thermal) losses to the wall $[25,29]$. The results included multi-front (spontaneous generation of transverse waves) detonation-like features in a high-speed phase, loss of these multifront features in a low-speed phase, and wave velocity oscillations reminiscent of galloping detonation, but with much lower amplitudes. However, the full range of behavior with pressure and the inclusion of all loss mechanisms have not yet been explored with direct numerical simulation due to the extreme requirements for spatial and temporal resolution necessary for resolving these low- and high-speed phases simultaneously. For this reason, simplified models such as those discussed by Ul'yanitskii [26] and Aksamentov et al. [28] still provide a useful framework for understanding the competition of friction and chemical reaction as well as the role of gas dynamics in these flows.

Both types of frictional effects depend strongly on the Reynolds number $R e=$ $\rho U \ell / \mu$, which determines both the flow regime (laminar or turbulent) and the magnitude of the displacement effect or friction factor, where $\rho, U, \ell$, and $\mu$ are the gas density, velocity, distance behind the shock, and dynamic viscosity, respectively. The pressure dependence enters primarily through $\rho$ with all other factors being the same, and thus $R e$ is proportional to the initial pressure. The various frictional effects depend on fractional powers of the Reynolds number, for example, the ratio of the boundary layer thickness to distance behind the shock is proportional to $R e^{-n}$ with $n=1 / 2$ for laminar flow and $n \approx 0.2$ to 0.3 for turbulent flow. The unit Reynolds numbers $R e^{\prime}=\rho U / \mu$ depend strongly on the gas temperature and to a lesser extent the shock speed. In completely reacted gas behind the front of a CJ detonation, $R e^{\prime}=8.5 \times 10^{6} \mathrm{~m}^{-1}$ for $P_{0}=20 \mathrm{kPa}$ and 3 $\times 10^{6} \mathrm{~m}^{-1}$ for $P_{0}=7 \mathrm{kPa}$; in the shocked but unreacted gas the values are nearly an order of magnitude larger: $R e^{\prime}=5.9 \times 10^{7} \mathrm{~m}^{-1}$ for $P_{0}=20 \mathrm{kPa}$ and $2 \times 10^{7}$ $\mathrm{m}^{-1}$ for $P_{0}=7 \mathrm{kPa}$. In both cases, the values of $R e^{\prime}$ are inversely proportional to pressure and the magnitudes are sufficiently large that transition to turbulence is expected within the first 0.2 to $0.5 \mathrm{~m}$ in the burned gas and the first 0.02 to $0.05 \mathrm{~m}$ of the shocked but unburned gas. These values need to be considered relative to the magnitude of the reaction zone thicknesses which are discussed next.

The chemical reaction rates and consequently the reaction times (induction 
and energy release) and length scales depend on pressure through the composition dependence of the reaction rate. For the propane-oxygen mixtures considered in the present study, reaction time and length scales have been estimated using a detailed chemical reaction mechanism (GRI-Mech 3.0) and simplified models of the combustion process. The idealized ZND model of detonation structure predicts that, at $20 \mathrm{kPa}$ initial pressure, a CJ detonation has an induction zone length that is $165 \mu \mathrm{m}$ and an energy release zone of $47 \mu \mathrm{m}$; at $7 \mathrm{kPa}$, the induction zone length is $465 \mu \mathrm{m}$ and the energy release zone is $145 \mu \mathrm{m}$. Typical of the high temperature (1800 to $3300 \mathrm{~K}$ in this case) conditions of fuel-oxygen detonations, these length scales are in ratio inversely proportional to pressure. The induction times exhibit a very strong dependence on initial shock strength and the energy release times are almost independent of the initial shock strength. As a consequence, in the phase of the galloping wave when the leading shock decays, reactions take place in progressively lower temperature and pressure environments, reducing the reaction rates in the induction zone and causing the reaction front to progressively lag behind the shock. However, once the induction phase has proceeded sufficiently to create a substantial pool of radicals and intermediates, the reactions rapidly go to completion, releasing energy quickly in the shocked but unreacted gases, consistent with the model of Ul'yanitskii [26].

The observed variations in luminous zone velocity distributions with initial pressure may originate from the increasing thickness of the boundary layer as compared to that of the reaction zone as the pressure decreases. Figure 15 is particularly remarkable with the change in the duration of the high-speed phase decreasing by a factor of 100 for an initial pressure change of only a factor of 3 . This strong sensitivity of the high-speed phase duration to the initial pressure may be particular to this mixture, which has a very high effective activation energy (about $40 \mathrm{kcal} / \mathrm{mole}$ for conditions representative of the CJ state) - a situation that is known to result in extreme sensitivity of detonation behavior to initial condition changes.

Early studies on one-dimensional detonation with multi-step kinetics [30-32] identified that detonations became increasingly unstable as the induction zone length grew relative to the energy release zone length and led to the identification of quantitative parameters proposed to govern stability [31,33]. The similar competition between the chemical and viscous- or thermal-loss length scales and their relative variations with pressure are likely relevant to the behavior exhibited in our stability analysis above (Sec. 4.5). For example, at certain pressures, distinct combinations of these length scales may couple to promote more periodic failure and reinitiation profiles, yielding the regular phase diagram behavior 
observed in Figs. $18(15 \mathrm{kPa})$ and $20(8 \mathrm{kPa})$ and the corresponding strong and isolated peaks in the bifurcation diagram of Fig. 22. Other length scale combinations may yield less regular or aperiodic behavior, as seen in the phase diagrams for $9.0 \mathrm{kPa}$ (Fig. 19) and $7.0 \mathrm{kPa}$ (Fig. 21), which generate diffuse distributions (characteristic of aperiodic motion) on the bifurcation diagram. The present wave dynamics exhibit a number of different propagation modes and are expected to depend on several nondimensional parameters, rather than a unique ratio as seen in 1D calculation $[31,33]$. Simplified or analog models may provide a computationally efficient approach to explore these wave dynamics.

\section{Conclusions}

A quantitative analysis of luminous front velocity characteristics versus initial mixture pressure was performed for near-limit detonation propagation in stoichiometric propane-oxygen mixtures confined in polyurethane tubing with the tube diameter on the order of the detonation cell size. The use of extremely long tube lengths (with $L / d>7,300$ ) allowed observation of up to 20 galloping cycles. The results showed that two dominant velocity modes exist near $0.4 D_{\mathrm{CJ}}$ and 0.95 $D_{\mathrm{CJ}}$, with the lower velocity mode becoming more prevalent with decreasing mixture initial pressure. The results of multiple experiments were used to generate a probability distribution function for velocity in order to visualize this behavior.

Analysis of the galloping frequency indicated a dominant frequency band between 0.8 and $1.0 \mathrm{kHz}$ that was not a strong function of initial pressure, consistent with the previous observations of the high-velocity phase being a detonation-like wave with a reaction zone extent determined by chemical reaction rates and the low-velocity phase being a complex of a shock and a premixed flame with propagation rates which are relatively independent of pressure. The oscillatory behavior appears to be consistent with a self-excited oscillation between the trailing reaction zone and the leading shock front that previous researchers have noted for galloping detonations. The observed frequency is inconsistent with both transverse and longitudinal modes associated with acoustic wave propagation within either products or reactants. (Estimates based on postshock sound speeds and the tube diameter indicate that the lowest transverse mode frequencies will be on the order of 100 to $250 \mathrm{kHz}$ while estimates based on the burned gas sound speed and length of the spiral predict longitudinal mode frequencies on the order of 10-30 Hz.)

Wave acceleration trajectories during the DDT and detonation failure phases of the galloping mode were repeatable and insensitive to variations in initial pres- 
sure. The amount of the time spent per galloping cycle in the low velocity mode was a weak function of mixture pressure, while the amount of time spent per cycle in the high velocity mode was a strong function of pressure. These results indicate that galloping detonation is a regular phenomenon that persists over extremely long tube lengths. The luminous front velocity-time series were analyzed using methods of dynamical systems. Phase plane and bifurcation diagrams show a sequence of changes with decreasing initial pressure that are typical of deterministic systems that exhibit transition to aperiodic orbits through a series of perioddoubling bifurcations, similar to that observed in idealized one-dimensional calculations without losses at the channel or tube boundaries. Based on the universality of the velocity behavior within a galloping cycle, we conjecture that the galloping detonation occurs in a regime where chemical reaction and wall confinement effects simultaneously promote the onset of DDT, while preventing steady detonation propagation. Thus, our results indicate that, in order to better predict this behavior, future research should work to elucidate the physical phenomena responsible for the specific failure and initiation mechanisms observed, as well as their variation with pressure and tube diameter. 


\section{Appendix A. Velocity Histories}
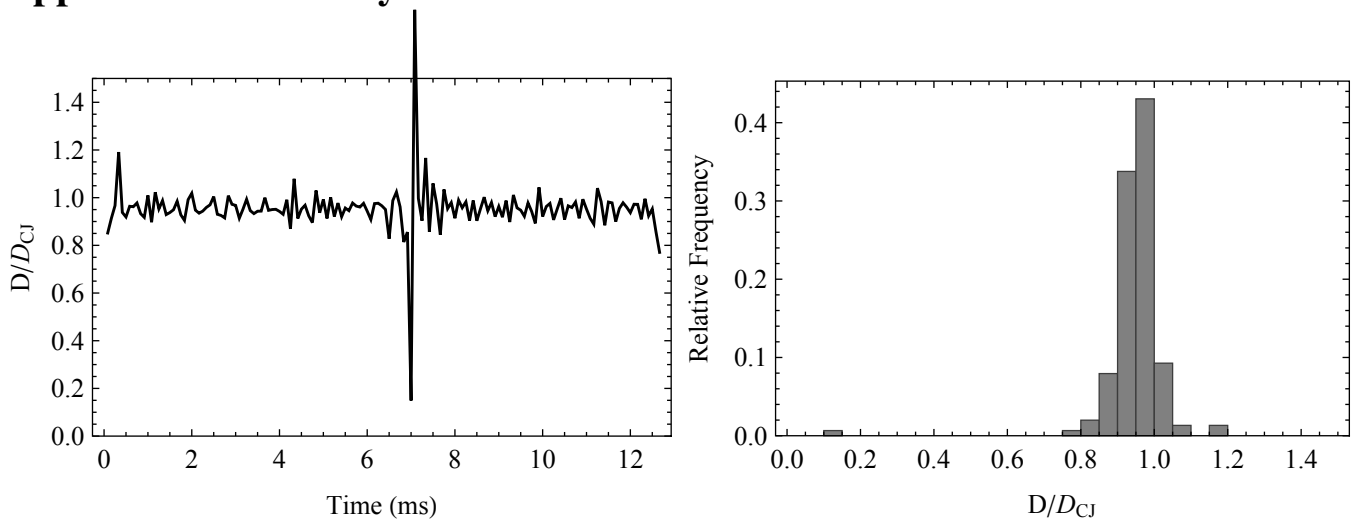

Figure A.23: Velocity history at $20.1 \mathrm{kPa}$ from test 190.
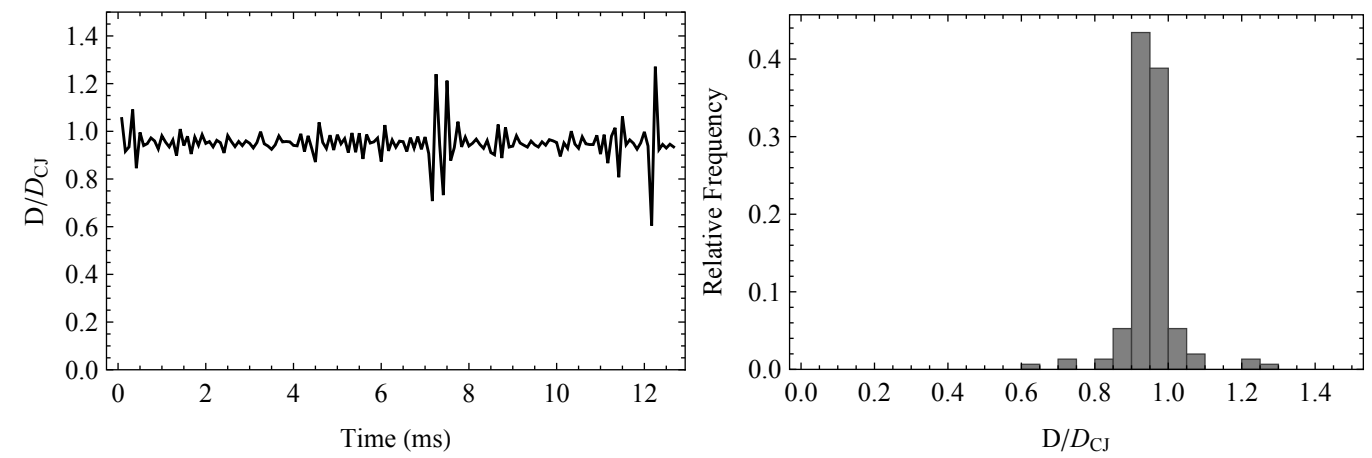

Figure A.24: Velocity history at $19.1 \mathrm{kPa}$ from test 189.
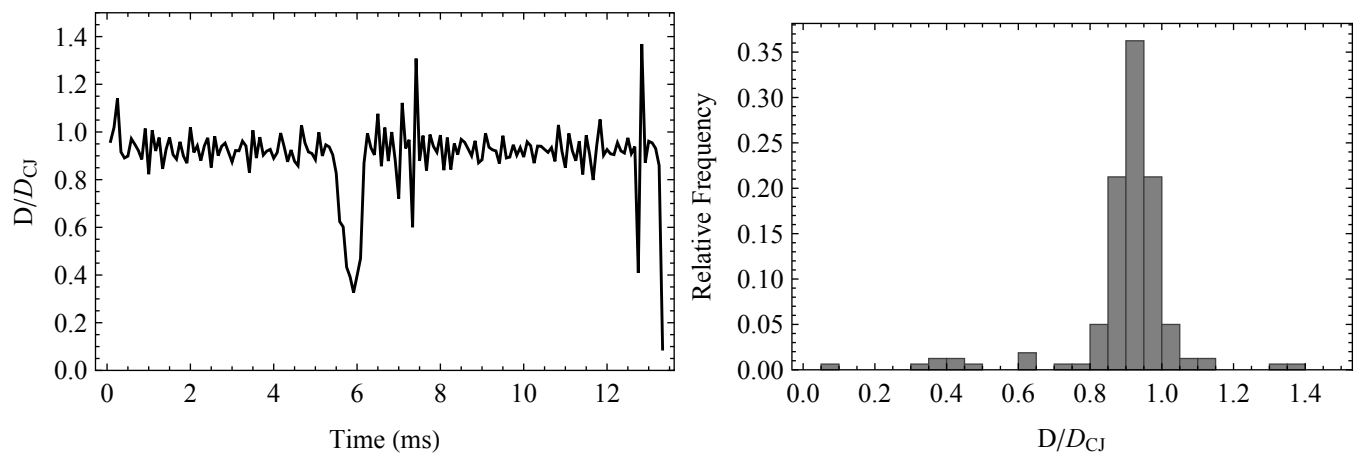

Figure A.25: Velocity history at $15.1 \mathrm{kPa}$ from test 191. 

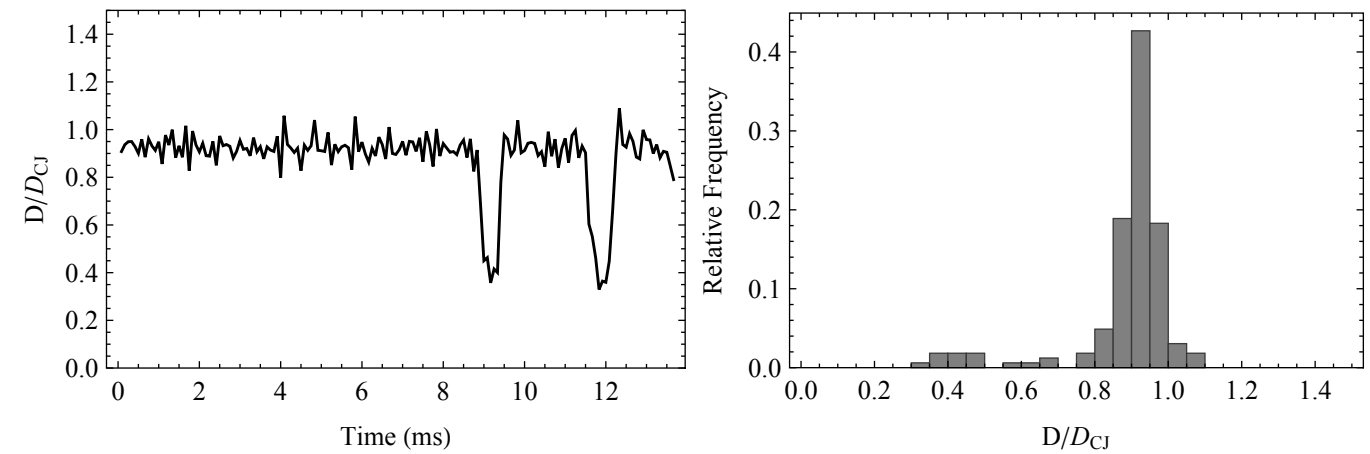

Figure A.26: Velocity history at $15.0 \mathrm{kPa}$ from test 195.
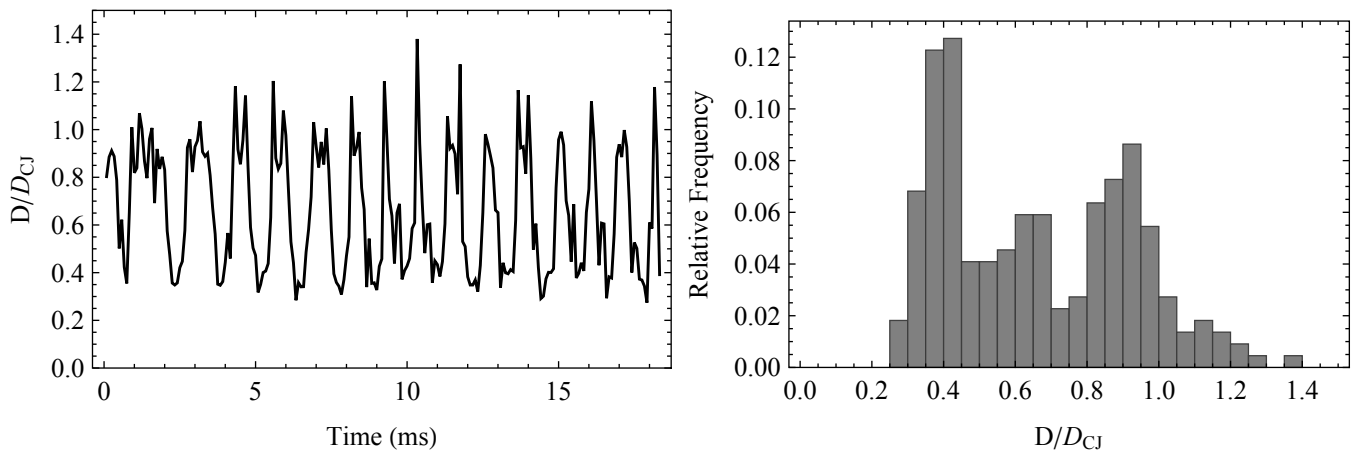

Figure A.27: Velocity history at $12.0 \mathrm{kPa}$ from test 192.
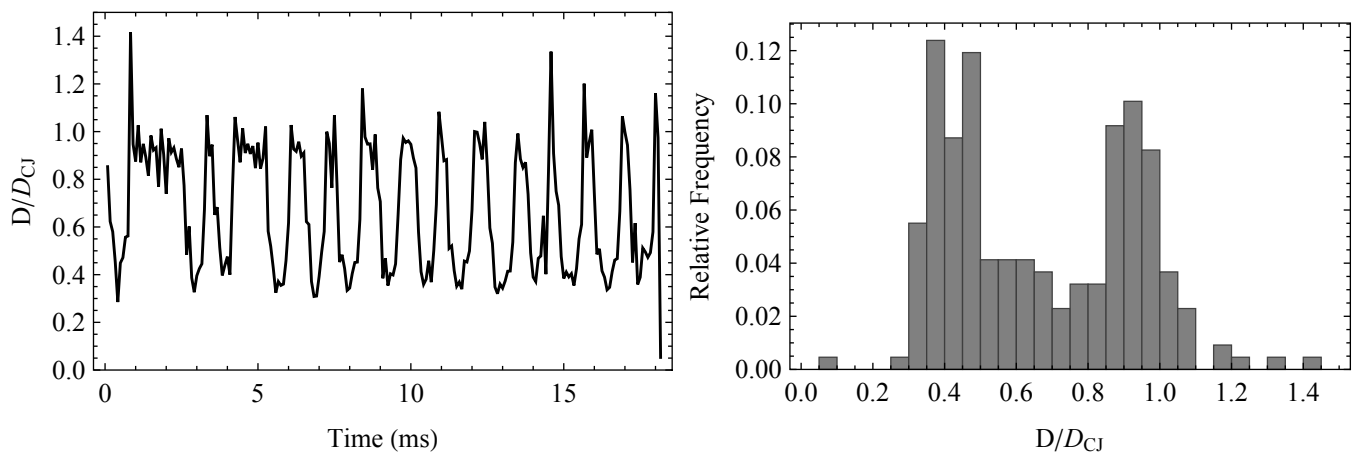

Figure A.28: Velocity history at $12.0 \mathrm{kPa}$ from test 198. 

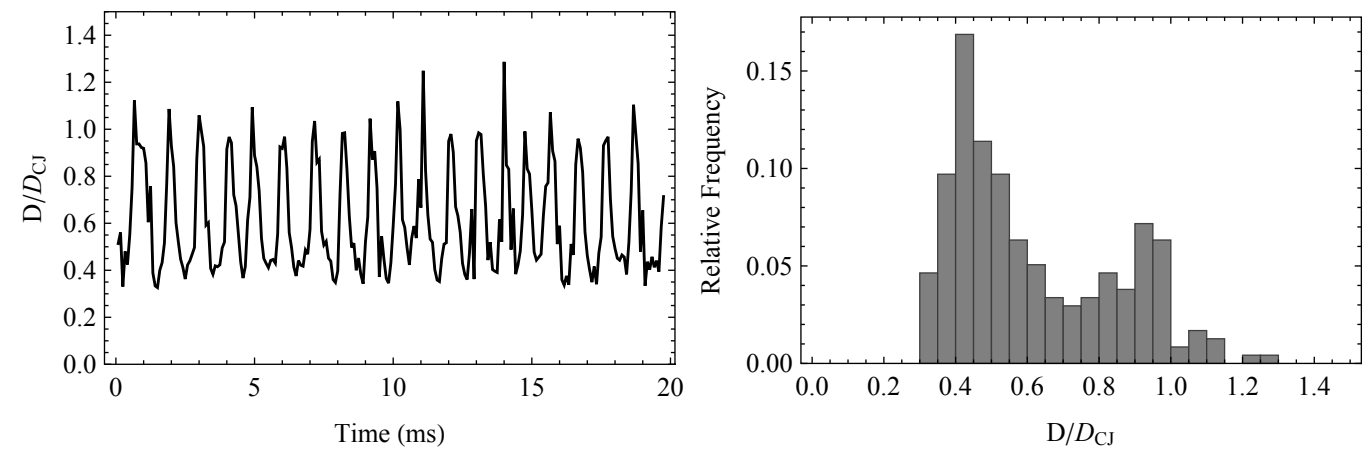

Figure A.29: Velocity history at $10.1 \mathrm{kPa}$ from test 194.
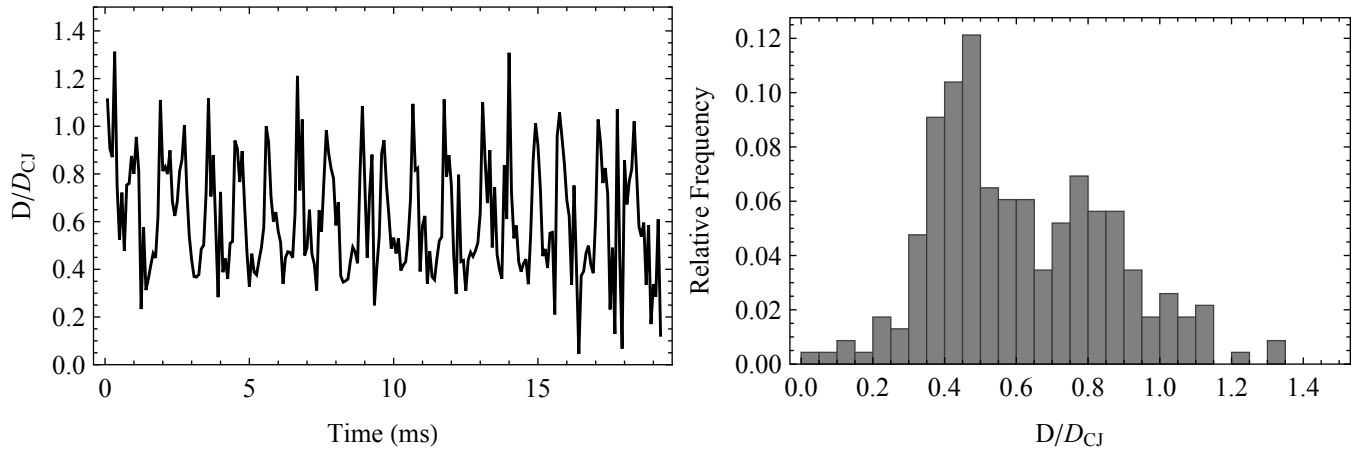

Figure A.30: Velocity history at $10.0 \mathrm{kPa}$ from test 193.
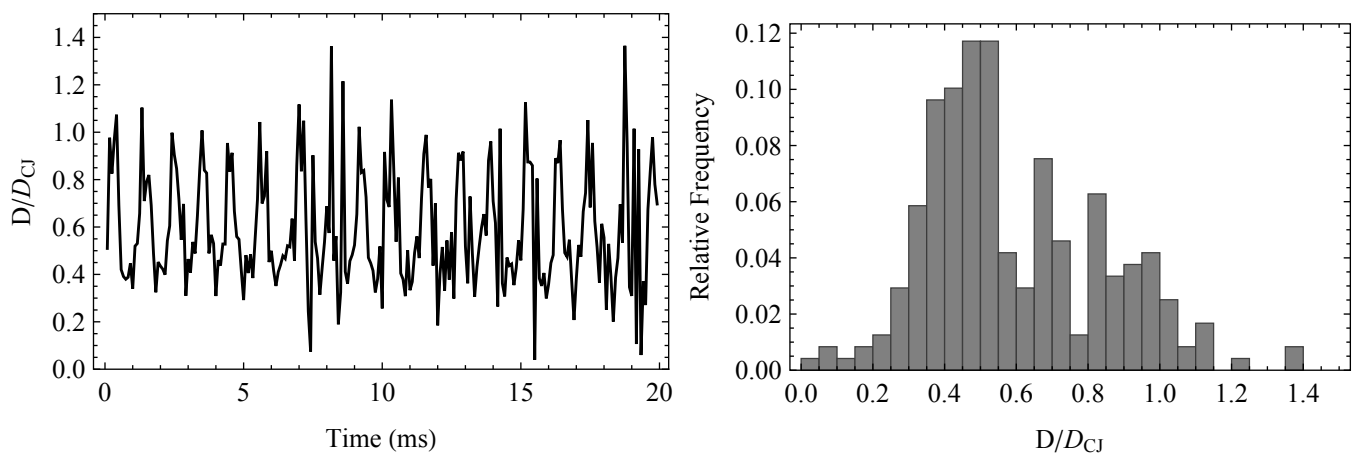

Figure A.31: Velocity history at $9.0 \mathrm{kPa}$ from test 202. 

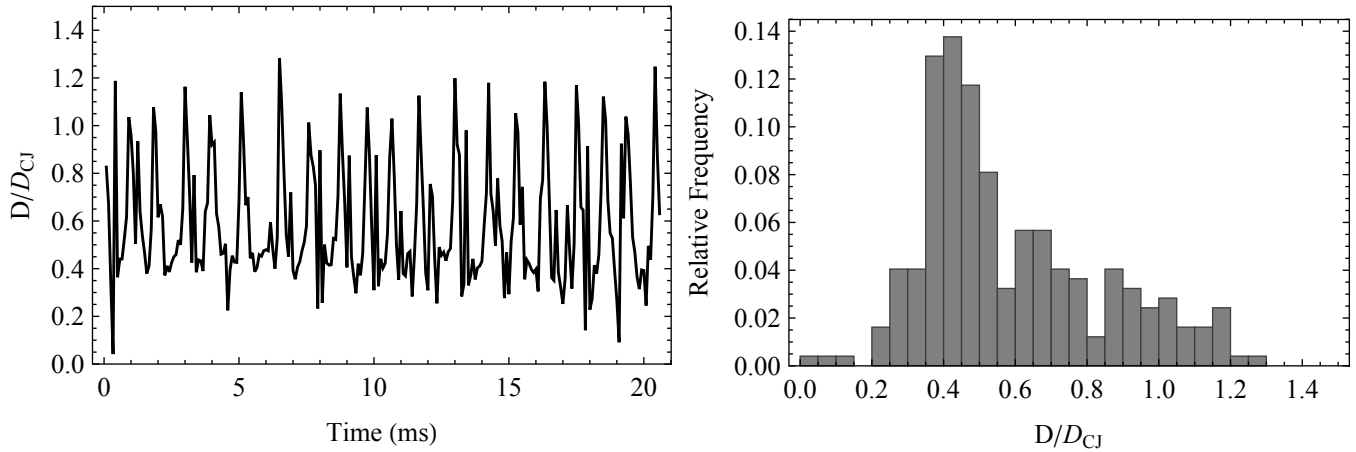

Figure A.32: Velocity history at $9.0 \mathrm{kPa}$ from test 203.
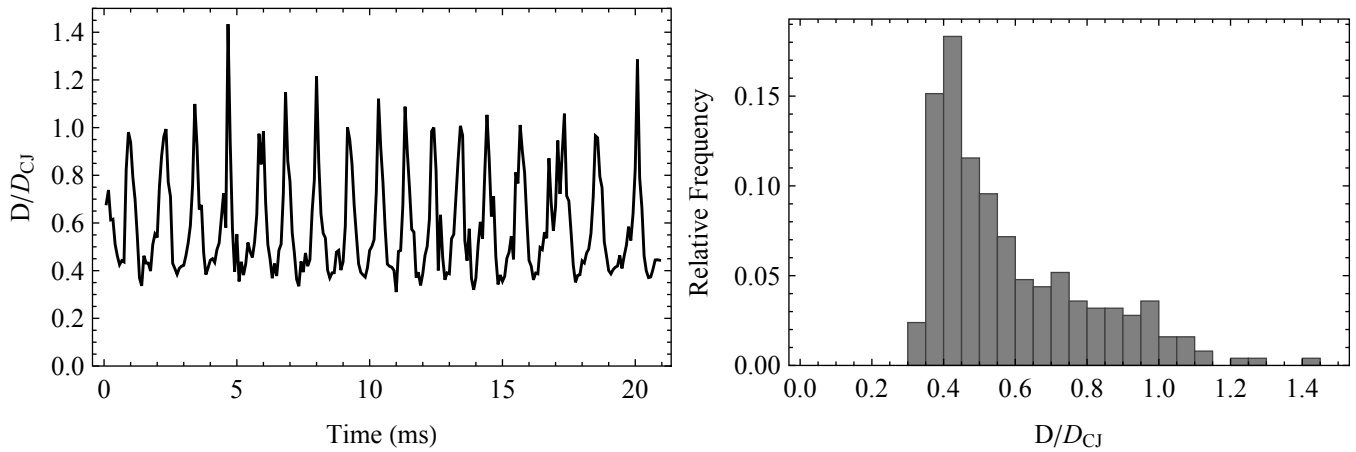

Figure A.33: Velocity history at $8.0 \mathrm{kPa}$ from test 196.
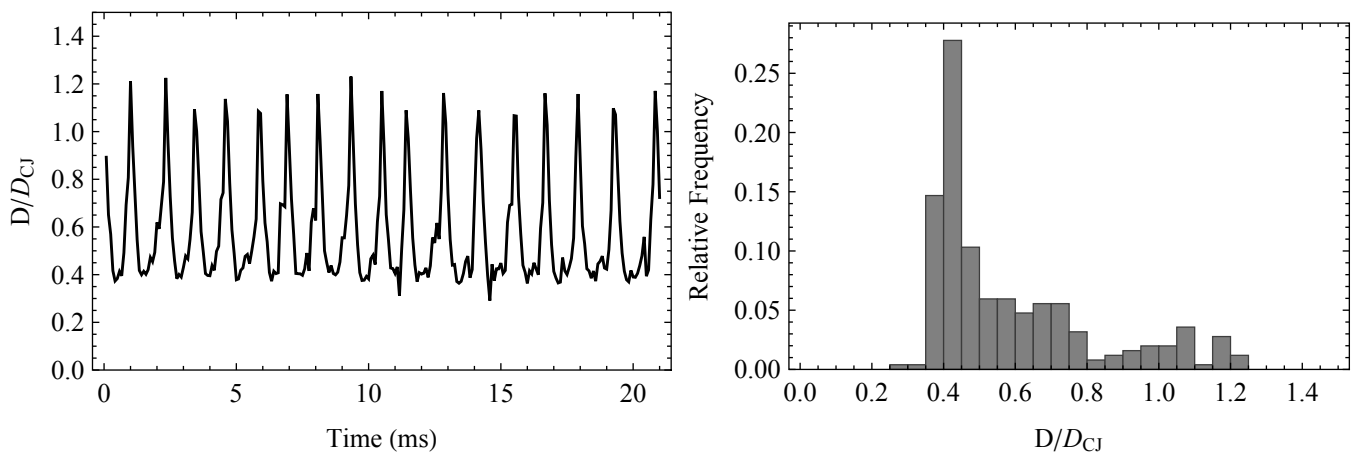

Figure A.34: Velocity history at $8.0 \mathrm{kPa}$ from test 197. 

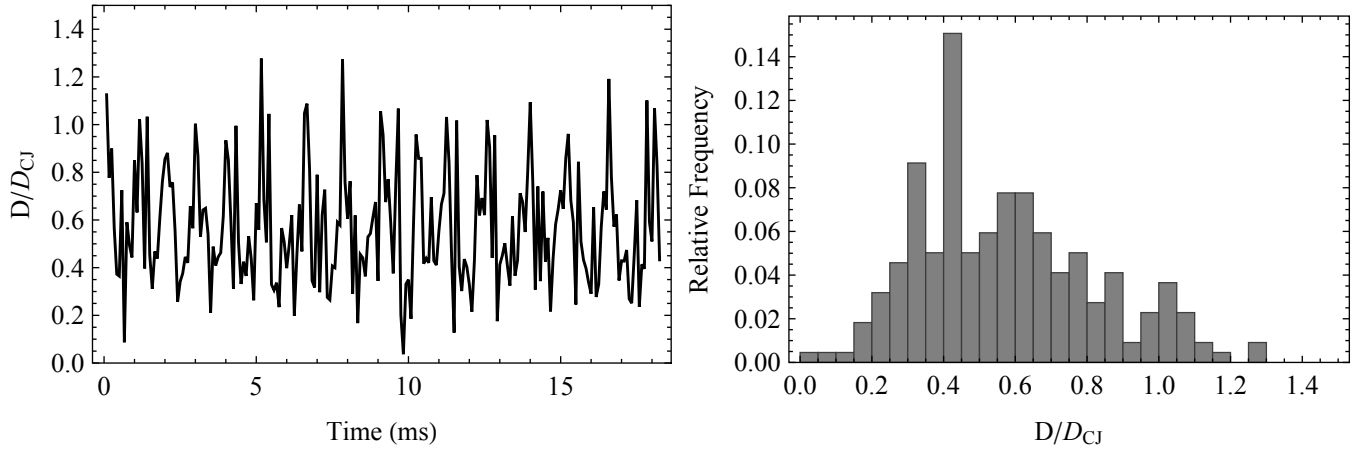

Figure A.35: Velocity history at $7.6 \mathrm{kPa}$ from test 199.
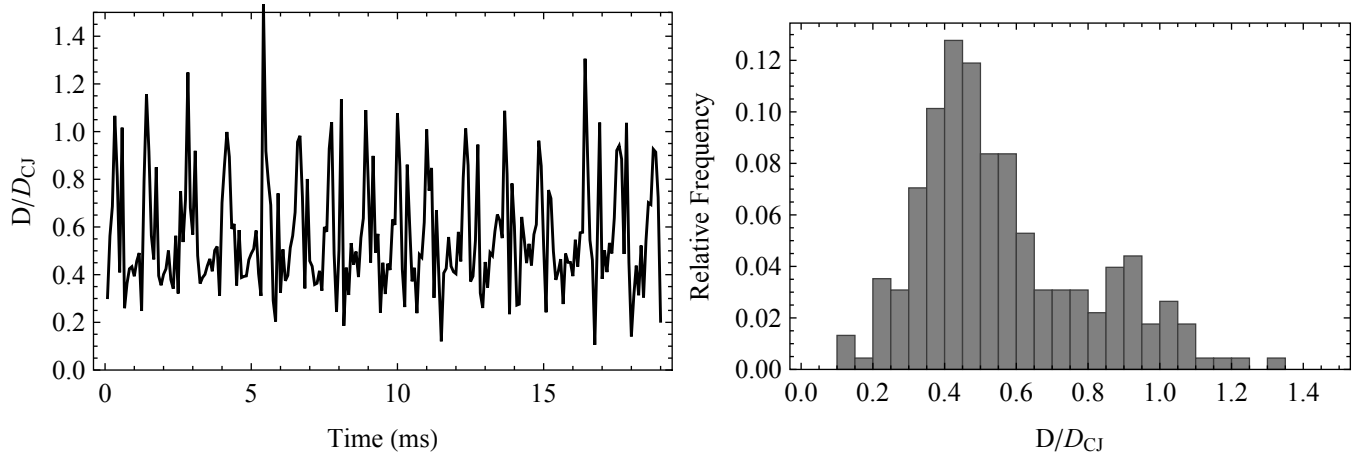

Figure A.36: Velocity history at $7.6 \mathrm{kPa}$ from test 204.
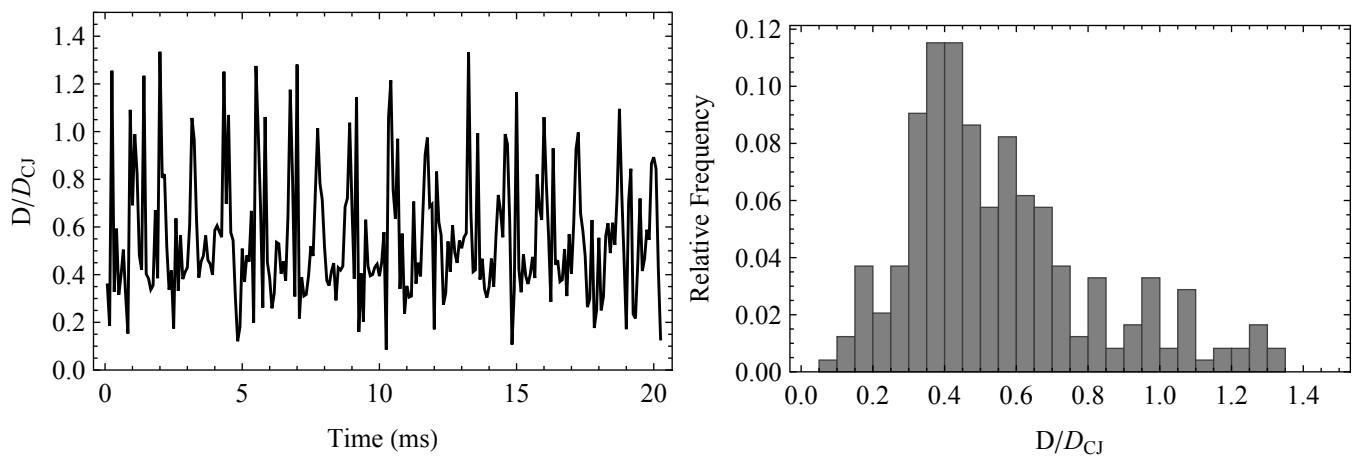

Figure A.37: Velocity history at $7.1 \mathrm{kPa}$ from test 200. 

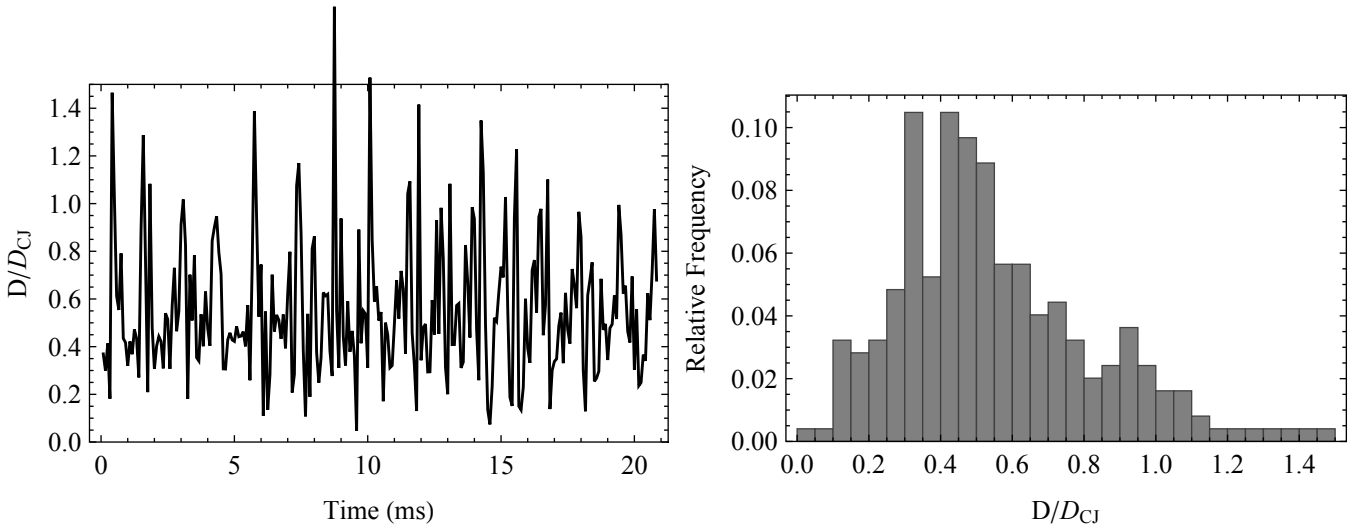

Figure A.38: Velocity history at $7.0 \mathrm{kPa}$ from test 201. 


\section{References}

[1] V. Manzhalei, Detonation of acetylene near the limit, Combustion, Explosion, and Shock Waves 11 (1975) 128-130.

[2] J. Lee, G. Dupre, R. Knystautas, L. Lee, Doppler interferometry study of unstable detonations, Shock Waves 5 (1995) 175-181.

[3] V. Manzhalei, Low-velocity detonation limits of gaseous mixtures, Combustion, Explosion, and Shock Waves 35 (1999) 296-302.

[4] F. Haloua, M. Brouillette, V. Lienhart, G. Dupre, Characteristics of unstable detonations near extinction limits, Combustion and Flame 122 (2000) 422 438.

[5] Y. Gao, J. Lee, H. Ng, Velocity fluctuation near the detonation limits, Combustion and Flame 128 (2014) 191-196.

[6] S. Jackson, Gaseous Detonation Initiation via Wave Implosion, Ph.D. thesis, California Institute of Technology, Pasadena, CA, 2005.

[7] A. Camargo, H. Ng, J. Chao, J. Lee, Propagation of near-limit gaseous detonations in small diameter tubes, Shock Waves 20 (2010) 499-508.

[8] W. Huang, Combustion modes in small tubes, 2005. SURF Project Report, California Institute of Technology, Pasadena CA.

[9] S. Jackson, B. Lee, W. Huang, F. Pintgen, J. Karnesky, Z. Liang, J. Shepherd, Experimental detonation propagation under high loss conditions, Proceedings of the 22nd International Colloquium on the Dynamics of Explosions and Reactive Systems (2009).

[10] D. Edwards, G. Hooper, J. Morgan, A study of unstable detonations using a microwave interferometer, Journal of Physics D - Applied Physics 7 (1971) 242-248.

[11] J. Austin, The Role of Instability in Gaseous Detonation, Ph.D. thesis, California Institute of Technology, Pasadena, CA, 2003.

[12] E. Schultz, J. Shepherd, Validation of detailed reaction mechanisms for detonation simulation, GALCIT Technical Report FM99-5, Graduate Aeronautical Laboratories, California Institute of Technology, Pasadena, CA, 2000. 
[13] M.-H. Wu, C.-Y. Wang, Reaction propagation modes in millimeter-scale tubes for ethylene/oxygen mixtures, Proceedings of the Combustion Institute 33 (2011) 2287-2293.

[14] M. Kaneshige, J. Shepherd, Detonation Database, Technical Report FM978, Graduate Aeronautical Laboratories, California Institute of Technology, Pasadena, CA, 1997.

[15] J. Anderson, Hypersonic and high temperature gas dynamics, McGraw-Hill, Inc., New York, 2000.

[16] J. Lee, The Detonation Phenomenon, Cambridge University Press, New York, 2008.

[17] F. Zhang, R. Chue, J. Lee, R. Klein, A nonlinear oscillator concept for onedimensional pulsating detonations, Shock Waves 8 (1998) 351-359.

[18] A. Kasimov, D. Stewart, On the dynamics of self-sustained one-dimensional detonations: A numerical study in the shock-attached frame, The Physics of Fluids 16 (2004) 3566-3578.

[19] H. Ng, A. Higgins, C. Kiyanda, M. Radulescu, J. Lee, K. Bates, N. Nikiforakis, Nonlinear dynamics and chaos analysis of one-dimensional pulsating detonations, Combustion Theory and Modelling 9 (2005) 159-170.

[20] A. Henrick, T. Aslam, J. Powers, Simulations of pulsating one-dimensional detonations with true fifth order accuracy, Journal of Computational Physics 213 (2006) 311-329.

[21] H. Abderrahmane, F. Paquet, H. Ng, Applying nonlinear dynamic theory to one-dimensional pulsating detonations, Combustion Theory and Modelling 15 (2011) 205-225.

[22] C. Romick, T. Aslam, J. Powers, The effect of diffusion on the dynamics of unsteady detonations, Journal of Fluid Mechanics 699 (2012) 453-464.

[23] A. Sow, A. Chinnayya, A. Hadjadj, Mean structure of one-dimensional unstable detonations with friction, Journal of Fluid Mechanics 743 (2014) 503-533.

[24] L. Massa, R. Kumar, P. Ravindran, Dynamic mode decomposition analysis of detonation waves, Physics of Fluids 24 (2012) 0166101. 
[25] N. Tsuboi, Y. Morii, A. Hayashi, Transient development of friction-induced low-velocity detonations, Proceedings of the Combustion Institute 28 (2000) 645-651.

[26] V. Ul'yanitskii, Galloping mode in a gas detonation, Combustion, Explosion, and Shock Waves 17 (1981) 93-97.

[27] J. Fay, Two-dimensional gaseous detonations: Velocity deficit, The Physics of Fluids 2 (1959) 283-289.

[28] S. Aksamentov, V. Manzhaley, V. Mitrofanov, Numerical modeling of galloping detonation, Progress in Astronautics and Aeronautics 153 (1993) $112-112$.

[29] A. Chinnayya, A. Hadjadj, D. Ngomo, Computational study of detonation wave propagation in a narrow channel, Physics of Fluids 25 (2011) 036101.

[30] M. Short, J. Quirk, On the nonlinear stability and detonability limit of a detonation wave for a model three-step chain-branching reaction, Journal of Fluid Mechanics 339 (1997) 89-119.

[31] M. Short, A nonlinear evolution equation for pulsating Chapman-Jouguet detonations with chain-branching kinetics, Journal of Fluid Mechanics 430 (2001) 381-400.

[32] M. Short, G. Sharpe, Pulsating instability of detonations with a two-step chain-branching reaction model: theory and numerics, Combustion Theory and Modelling 7 (2003) 401-416.

[33] H. Ng, M. Radulescu, A. Higgins, N. Nikiforakis, J. Lee, Numerical investigation of the instability for one-dimensional Chapman-Jouguet detonations with chain-branching kinetics, Combustion Theory and Modelling 9 (2005) 385-401. 